\title{
On definability in some lattices of semigroup varieties
}

\author{
Mariusz Grech • Olga Sapir
}

Dedicated to the memory of Jaroslav Ježek.

Received: 28 January 2011 / Accepted: 13 August 2012 / Published online: 11 September 2012

(C) The Author(s) 2012. This article is published with open access at Springerlink.com

\begin{abstract}
An identity of the form $x_{1} \cdots x_{n} \approx x_{1 \pi} x_{2 \pi} \cdots x_{n \pi}$ where $\pi$ is a non-trivial permutation on the set $\{1, \ldots, n\}$ is called a permutation identity. If $u \approx v$ is a permutation identity, then $\ell(u \approx v)$ [respectively $r(u \approx v)$ ] is the maximal length of the common prefix [suffix] of the words $u$ and $v$. A variety that satisfies a permutation identity is called permutative. If $\mathcal{V}$ is a permutative variety, then $\ell=\ell(\mathcal{V})$ [respectively $r=r(\mathcal{V})$ ] is the least $\ell$ [respectively $r$ ] such that $\mathcal{V}$ satisfies a permutation identity $\tau$ with $\ell(\tau)=\ell$ [respectively $r(\tau)=r$ ]. A variety that consists of nil-semigroups is called a nil-variety. If $\Sigma$ is a set of identities, then $\operatorname{var} \Sigma$ denotes the variety of semigroups defined by $\Sigma$. If $\mathcal{V}$ is a variety, then $L(\mathcal{V})$ denotes the lattice of all subvarieties of $\mathcal{V}$.

For $\ell, r \geq 0$ and $n>1$ let $\mathfrak{B}_{\ell, r, n}$ denote the set that consists of $n$ ! identities of the form

$$
t_{1} \cdots t_{\ell} x_{1} x_{2} \cdots x_{n} z_{1} \cdots z_{r} \approx t_{1} \cdots t_{\ell} x_{1 \pi} x_{2 \pi} \cdots x_{n \pi} z_{1} \cdots z_{r},
$$

where $\pi$ is a permutation on the set $\{1, \ldots, n\}$. We prove that for each permutative nilvariety $\mathcal{V}$ and each $\ell \geq \ell(\mathcal{V})$ and $r \geq r(\mathcal{V})$ there exists $n>1$ such that $\mathcal{V}$ is definable by a first-order formula in $L\left(\operatorname{var} \mathfrak{B}_{l, r, n}\right)$ if $\ell \neq r$ or $\mathcal{V}$ is definable up to duality in $L\left(\operatorname{var} \mathfrak{B}_{\ell, r, n}\right)$ if $\ell=r$.
\end{abstract}

Keywords Permutation identity $\cdot$ Permutative variety $\cdot 0$-Reduced identity · Nil-variety · Lattice of subvarieties · First order definability

Communicated by Mikhail Volkov.

M. Grech was partially sponsored by Polish grant MNiSW N201 543038.

M. Grech (凷

Institute of Mathematics, University of Wrocław, pl. Grunwaldzki 2, 50-384 Wrocław, Poland e-mail: Mariusz.Grech@math.uni.wroc.pl

O. Sapir

Department of Mathematics, Vanderbilt University, Nashville, TN 37240, USA

e-mail: olga.sapir@gmail.com 


\section{Introduction}

A subset $A$ of a lattice $\langle L, \vee, \wedge\rangle$ is called definable in $L$ if there exists a first-order formula $\Phi(\bar{x})$ with one free variable $\bar{x}$ in the language of lattice operations $\vee$ and $\wedge$ that defines $A$ in $L$, that is, $\Phi(\bar{x})$ is true if and only if $\bar{x} \in A$. An element $a \in L$ is called definable in $L$ if the set $\{a\}$ is definable in $L$.

In [13, 22], Tarski and McKenzie raised problems of first-order definability in lattices of varieties of algebras. In a series of papers [5-8], Ježek solved the most general problems in this area for the lattice $\mathbf{L}_{T}$ of all varieties of algebras of any given type $T$. He proved that each finitely generated and each finitely based variety is definable in $\mathbf{L}_{T}$ up to the obvious, syntactically defined automorphisms, and consequently, $\mathbf{L}_{T}$ has no other automorphisms. He also proved that the set of all finitely generated varieties, the set of all one-based varieties, and the set of all finitely based varieties are definable in $\mathbf{L}_{T}$.

The results in [5-8] do not imply that the same would be true for the lattice of subvarieties of a given variety, but they suggest that the same technique could be used in the cases when the variety is defined by linear identities. Recall that an identity $u \approx v$ is called linear if each variable occurs once in $u$ and once in $v$.

If $\Sigma$ is a set of identities, then $\operatorname{var} \Sigma$ denotes the variety defined by $\Sigma$. If $\mathcal{V}$ is a variety, then $L(\mathcal{V})$ denotes the lattice of all subvarieties of $\mathcal{V}$. Ježek and McKenzie raised problems of first-order definability in lattices of the form $L(\operatorname{var} \mathfrak{B})$ where $\mathfrak{B}$ is a set of linear identities. In the case of groupoids, the most significant lattices of this form are the lattice of all semigroup varieties $\mathbf{S E M}=L(\operatorname{var}\{x(y z) \approx(x y) z\})$, the lattice of all varieties of commutative groupoids $\mathbf{G C O M}=L(\operatorname{var}\{x y \approx y x\})$ and the lattice of all varieties of commutative semigroups $\mathbf{C O M}=L(\operatorname{var}\{x(y z) \approx$ $(x y) z, x y \approx y x\})$.

In [11], Ježek and McKenzie adapting the approach used in [5-8] proved that several important sets of semigroup varieties such as the sets of all finitely generated, locally finite and finitely based varieties are definable in SEM. Moreover, they proved that each finitely generated and each finitely based locally finite variety is individually definable up to duality (i.e. up to inverting the order of occurrence of letters in defining identities). They conjectured that the local finiteness assumption in the last quoted result may be omitted and that, in consequence, the lattice SEM has no nontrivial automorphisms except duality, but this conjecture still remains unproven.

In [12], Kisielewicz adapting the approach used in [11] proved that many sets and individual varieties are definable in COM. However, he discovered that there exist nontrivial automorphisms of COM. In [2], the first-named author of the present paper completed the study of first-order definability in COM. He obtained complete descriptions of the set of definable varieties and of the group of automorphisms of COM, which turned out to be an uncountable Boolean group.

In [9, 10], Ježek obtained definability for some broad classes of varieties in GCOM and conjectured that the lattice GCOM has no nontrivial automorphisms.

It seems that having both the associativity and the commutativity identities in $\mathfrak{B}$ makes the study of definability in lattices of the form $L(\operatorname{var} \mathfrak{B})$ much easier than having only associativity (SEM) or only commutativity (GCOM). The commutativity 
identity $x y \approx y x$ is the strongest type of a permutation identity, i.e. a linear identity that is non-trivial modulo $x(y z) \approx(x y) z$. In [3], the first-named author took the next step up from the case of COM to a more difficult case when the set $\mathfrak{B}$ contains the associativity identity and some permutation identities.

For the rest of the article we consider only subvarieties of SEM. An identity $u \approx v$ is called balanced if each variable occurs the same number of times in $u$ and $v$. If $u \approx v$ is a balanced identity, then $\ell(u \approx v)$ [respectively $r(u \approx v)$ ] is the maximal length of the common prefix [suffix] of the words $u$ and $v$. A variety that satisfies a permutation identity is called permutative. If $\mathcal{V}$ is a permutative variety, then $\ell=$ $\ell(\mathcal{V})$ [respectively $r=r(\mathcal{V})$ ] is the least $\ell$ [respectively $r$ ] such that $\mathcal{V}$ satisfies a permutation identity $\tau$ with $\ell(\tau)=\ell$ [respectively $r(\tau)=r$ ]. For each $k \geq 0$, a variety $\mathcal{V}$ is called $k$-permutative if $\ell(\tau) \leq k$ and $r(\tau) \leq k$. (In view of Lemma 11.2 this definition is equivalent to the definition of a $k$-permutative variety given in [17].) For each $k \geq 0$ the set $\mathbf{P}(\mathbf{k})$ of all $k$-permutative varieties forms a sublattice of the lattice of all permutative varieties $\mathbf{P}$. It is easy to see that the lattice $\mathbf{P}(\mathbf{0})$ contains $\mathbf{C O M}$ and for each $k \geq 0$ we have that $\mathbf{P}(\mathbf{k}) \subset \mathbf{P}(\mathbf{k}+\mathbf{1})$. Since every permutative variety is $k$-permutative for some $k=0,1, \ldots, c$, the lattice $\mathbf{P}$ is the union of all the lattices $\mathbf{P}(\mathbf{k}), k=0,1, \ldots, c$.

Let $\delta: \mathcal{V} \mapsto \mathcal{V}^{\delta}$ denote the duality automorphism of SEM. We say that a lattice $\mathbf{L}$ of semigroup varieties is self-dual if $\mathbf{L}^{\delta}=\mathbf{L}$. If $\mathbf{L}$ is a self-dual sublattice of $\mathbf{S E M}$ then we say that a variety $\mathcal{V} \in \mathbf{L}$ is semi-definable in $\mathbf{L}$ if the set $\left\{\mathcal{V}, \mathcal{V}^{\delta}\right\}$ is definable in $\mathbf{L}$. In [3], the first-named author proved that each 0-permutative variety is semidefinable in $\mathbf{P}(\mathbf{0})$ and consequently, the lattice $\mathbf{P}(\mathbf{0})$ has no nontrivial automorphisms except $\delta$.

For $\ell, r \geq 0$ and $n>1$ let $\mathfrak{B}_{\ell, r, n}$ denote the set that consists of $n$ ! identities of the form $t_{1} \cdots t_{\ell} x_{1} x_{2} \cdots x_{n} z_{1} \cdots z_{r} \approx t_{1} \cdots t_{\ell} x_{1 \pi} x_{2 \pi} \cdots x_{n \pi} z_{1} \cdots z_{r}$, where $\pi$ is a permutation on the set $\{1, \ldots, n\}$. It follows from a result of Putcha and Yaqub [16] (see Lemma 11.2) that for each permutative variety $\mathcal{V}$ and each pair of numbers $(\ell, r)$ with $\ell \geq \ell(\mathcal{V})$ and $r \geq r(\mathcal{V})$ one can find $n>1$ such that $\mathcal{V} \in L\left(\operatorname{var} \mathfrak{B}_{\ell, r, n}\right)$. In particular, each 0 -permutative variety is contained in $L\left(\operatorname{var} \mathfrak{B}_{0,0, n}\right.$ ) for some $n>1$. The most difficult part of proving [3, Theorem 10.9] that each 0-permutative variety is semi-definable in $\mathbf{P}(\mathbf{0})$ was proving that each 0 -permutative variety is semi-definable in $L\left(\operatorname{var} \mathfrak{B}_{0,0, n}\right)$ for some $n$ big enough.

If $u$ is a word and $x$ is a variable that does not occur in $u$, then the pair of identities $u x \approx x u \approx u$ is usually written as $u \approx 0$ and is referred to as a 0-reduced identity. A semigroup [respectively variety] that satisfies a 0-reduced identity is called a nilsemigroup [nil-variety]. It has been observed by many authors that solving a certain problem for nil-varieties in a given class of varieties is often crucial to solving the problem for the whole class. In this article, we prove (Theorem 11.1) that for each permutative nil-variety $\mathcal{V}$ and each $\ell \geq \ell(\mathcal{V})$ and $r \geq r(\mathcal{V})$ there exists $n>1$ such that $\mathcal{V}$ is definable in $L\left(\operatorname{var} \mathfrak{B}_{\ell, r, n}\right)$ if $\ell \neq r$ or $\mathcal{V}$ is semi-definable in $L\left(\operatorname{var} \mathfrak{B}_{\ell, r, n}\right)$ if $\ell=r$.

A variety that can be defined by 0 -reduced identities only is called 0 -reduced variety. A nil-variety is called $b 0$-reduced if whenever it satisfies an unbalanced identity $u \approx v$, it also satisfies the identity $u \approx 0$ (and consequently, $v \approx 0$ ). If $m>0$ and 
$d>0$, then $\mathbf{C}_{m, d}$ denotes the cyclic monoid $\left\langle a \mid a^{m}=a^{m+d}\right\rangle$. If $d>0$, then $\mathbf{C}_{0, d}$ denotes the cyclic group of order $d$. Note that $\mathbf{C}_{1,1}$ is the two-element semilattice and $\mathbf{C}_{0,1}$ is the trivial group.

Let I denote an ideal of SEM that contains $\operatorname{var}\{x y \approx y x\}$. It follows from [12] and [25] that for each $m \geq 0$ and $d>0$ the variety $\operatorname{var} \mathbf{C}_{m, d}$ is definable in $\mathbf{I}$ (see Lemmas 2.2 and 2.3). It has been proved in [25] that the class of all nil-varieties is definable in SEM. The same arguments can be used to show that the class of all nilvarieties in $\mathbf{I}$ is definable in $\mathbf{I}$ (see Lemma 2.4). By using an idea from [3], a result from [23] and some functions on varieties, we prove (Proposition 4.1) that a nilvariety $\mathcal{V}$ is $b 0$-reduced if and only if for each prime $d>1$, the variety $\mathcal{V} \vee \operatorname{var} \mathbf{C}_{0, d}$ is a cover of the variety $\mathcal{V}$. This implies (Theorem 4.1) that the set of all $b 0$-reduced varieties in $\mathbf{I}$ is definable in $\mathbf{I}$. This statement plays a similar role in our article as Theorem 1.11 in [11]. That theorem says that the set of all 0-reduced varieties is definable in SEM.

Let $\mathfrak{B}$ denote a set of balanced identities that contains a permutation identity. By using an idea from [3, Sect. 9] and a construction from [11] we prove (Proposition 8.2) that a $b 0$-reduced variety $\mathcal{V} \in L(\operatorname{var} \mathfrak{B})$ has exactly one cover in the class of all nil-varieties in $L(\operatorname{var} \mathfrak{B})$ if and only if $\mathcal{V}=\operatorname{var}\{u \approx 0, \mathfrak{B}\}$ for some word $u$. This implies (Theorem 8.1) that the set of all varieties of the form $\operatorname{var}\{u \approx 0, \mathfrak{B}\}$ is definable in $L(\operatorname{var} \mathfrak{B})$.

An identity $u \approx v$ is called regular if each variable that occurs in $u$ also occurs in $v$ and visa versa. In Sect. 5, with each set of regular identities $\mathfrak{B}$ we associate a quasi-order on the free semigroup by saying that $v \leq_{\mathfrak{B}} u$ if and only if $\operatorname{var}\{v \approx 0, \mathfrak{B}\} \mid=u \approx 0$. It is easy to see that the set of all varieties of the form $\operatorname{var}\{u \approx 0, \mathfrak{B}\}$ ordered under inclusion can be identified with the set $F_{\infty} / \Leftrightarrow \mathfrak{B}$ of equivalence classes of words ordered under $\leq_{\mathfrak{B}}$. We call these equivalence classes word patterns modulo $\mathfrak{B}$ because the set $F_{\infty} / \Leftrightarrow_{x} \approx x$ is precisely the ordered set of word patterns considered in [11, Sect. 2].

Based on the proof of [3, Theorem 9.3], we show (Corollaries 6.1 and 6.2) that for each $\ell, r \geq 0$ and $n>1$ each word pattern is semi-definable in $F_{\infty} / \Leftrightarrow_{\mathfrak{B}_{\ell, r, n}}$ if $\ell=r$ and is definable in $F_{\infty} / \Leftrightarrow_{\mathfrak{B}_{\ell, r, n}}$ if $\ell \neq r$. Corollaries 6.1, 6.2 and Theorem 8.1 imply (Theorem 9.1) that for each $\ell, r \geq 0$ and $n>1$, each variety of the form $\operatorname{var}\{u \approx 0$, $\left.\mathfrak{B}_{\ell, r, n}\right\}$ is semi-definable in $L\left(\operatorname{var} \mathfrak{B}_{\ell, r, n}\right)$ if $\ell=r$ and is definable in $L\left(\operatorname{var} \mathfrak{B}_{\ell, r, n}\right)$ if $\ell \neq r$.

In Sect. 10 , we consider varieties of the form $\operatorname{var}\left\{u \approx v, \mathfrak{B}_{\ell, r, n}\right\}$ where $u \approx v$ is a regular identity such that the words $u$ and $v$ are either equivalent or incomparable in the order $\leq_{\mathfrak{B}_{\ell, r, n}}$ and their lengths are shorter than $n+\ell+r$. By translating and generalizing methods used in $[2,3,12]$ we prove (Theorems 10.1 and 10.2) that each variety of this form is semi-definable in $L\left(\operatorname{var} \mathfrak{B}_{\ell, r, n}\right)$ if $\ell=r$ and definable in $L\left(\operatorname{var} \mathfrak{B}_{\ell, r, n}\right)$ if $\ell \neq r$.

Using Theorems 10.1, 10.2, 11.1 and the result of Volkov [28] (see Lemma 11.4) that each 0-permutative variety is a join of a nil-variety and a variety generated by a cyclic monoid (or group), we reprove [3, Theorem 10.9] that says that each 0 -permutative variety is semi-definable in $\mathbf{P}(\mathbf{0})$ (see Corollary 11.1). As another consequence of Theorem 11.1 we show (Corollary 11.2) that for each $0 \leq k \leq 2$, every $k$-permutative nil-variety is semi-definable in the lattice $\mathbf{P}(\mathbf{k})$ of all $k$-permutative varieties. 


\section{Some definable varieties and classes of varieties in I}

In this section we consider definability in a sublattice I of SEM that contains $\operatorname{var}\{x y \approx y x\}$ and also contains $L(\mathcal{V})$ whenever $\mathcal{V} \in \mathbf{I}$. In particular, I can be $L(\operatorname{var} \mathfrak{B})$ for some set of balanced identities $\mathfrak{B}$, or the lattice of all $k$-permutative varieties $\mathbf{P}(\mathbf{k})$ for some $k \geq 0$ or the lattice of all permutative varieties $\mathbf{P}$.

Let $\mathcal{Z M}$ denote the variety $\operatorname{var}\{x y \approx 0\}$ of all semigroups with zero multiplication and $\mathcal{S} \mathcal{L}$ denote the variety of all semilattices var $\mathbf{C}_{1,1}$. Let $\mathbf{A}$ denote the set $\left\{\operatorname{var} \mathbf{C}_{0, d} \mid d\right.$ is prime $\}$ of all varieties of Abelian groups of prime exponents.

It is well-known (see [1], for example) that the set $\mathbf{A} \cup\{\mathcal{S} \mathcal{L}, \mathcal{Z} \mathcal{M}\}$ is the set of all atoms in $\mathbf{C O M}$ and that $\mathbf{I}$ may contain some of the two additional atoms of SEM, namely $\mathcal{L} \mathcal{Z}=\operatorname{var}\{x y \approx x\}$ and $\mathcal{R} \mathcal{Z}=\operatorname{var}\{x y \approx y\}$.

The following lemma is proved in [12, Proposition 3.1].

\section{Lemma 2.1 [12] The set $\mathbf{A}$ of all group atoms is definable in COM.}

Proposition 1.4 in [25] contains a simple explanation of how to tell apart a variety in $\mathbf{A}$ from $\mathcal{S} \mathcal{L}$ and from $\mathcal{Z} \mathcal{M}$ in SEM. The same explanation can be used to distinguish each variety in $\mathbf{A}$ from $\mathcal{S} \mathcal{L}$ and from $\mathcal{Z} \mathcal{M}$ in $\mathbf{C O M}$. According to [25, Proposition 1.4], for each prime $d>1$ the variety $\operatorname{var} \mathbf{C}_{0, d}$ is a proper subvariety of some commutative chain variety. The variety $\mathcal{Z} \mathcal{M}$ is also a proper subvariety of some commutative chain variety, but $\mathcal{S} \mathcal{L}$ is not properly contained in any chain variety. Also according to [29], the variety $\mathcal{Z M}$ is neutral in SEM (and, therefore, in COM) but the variety $\operatorname{var} \mathbf{C}_{0, d}$ is not neutral in COM for any $d>1[18,19]$.

The next lemma follows from Propositions 4.1 and 4.2 in [12].

Lemma 2.2 For each $m \geq 0$ and $d>0$, the variety $\operatorname{var} \mathbf{C}_{m, d}$ is definable in $\mathbf{C O M}$.

Corollary 4.8 in [25] contains an explicit first-order formula that defines the variety $\operatorname{var} \mathbf{C}_{m, d}$ for each $0 \leq m \leq \infty$ and $0<d \leq \infty$ in SEM. The same formula can be used to define var $\mathbf{C}_{m, d}$ in $\mathbf{C O M}$ (see [26]).

It is well known that the variety of all commutative semigroups is definable in $\mathbf{I}$ as the minimal variety in I that contains all group atoms. This gives us the following lemma.

Lemma 2.3 The variety of all commutative semigroups and the lattice of all commutative varieties COM are definable in $\mathbf{I}$.

The article [25] contains simple formulas that define many other well-known semigroup varieties and classes of varieties in SEM. The same formulas can be used to define these varieties and classes in $\mathbf{I}$.

Lemma 2.4 If $\mathbf{N}$ is the class of all nil-varieties in $\mathbf{S E M}$, then the class $\mathbf{I} \cap \mathbf{N}$ of all nil-varieties in $\mathbf{I}$ is definable in $\mathbf{I}$.

Proof Here are the two parts of the defining sentence of Vernikov [25, Theorem 2.2] formulated for $\mathbf{I}$. 
1. The variety of all semigroups with zero multiplication $\mathcal{Z} \mathcal{M}$ is the only neutral atom in $\mathbf{I}$ that is a proper subvariety of some chain variety.

2. $\mathbf{I} \cap \mathbf{N}$ is the class of all varieties in $\mathbf{I}$ that do not contain any atoms of $\mathbf{I}$ but $\mathcal{Z} \mathcal{M}$. (An explanation of this part can be found in [21] for instance).

The details on this defining sentence can be found in [25].

The next lemma follows from [12, Theorem 4.3] and can be proved exactly like [25, Proposition 1.7].

Lemma 2.5 For every $k>0$, the variety $\mathcal{N}_{k}=\operatorname{var}\left\{x^{2} \approx x_{1} x_{2} \cdots x_{k} \approx 0, x y \approx y x\right\}$ is definable in COM.

Lemma 2.6 The variety $\operatorname{var}\left\{x_{1} x_{2} \cdots x_{n} \approx 0\right\}$ is definable in $\mathbf{I}$ for each $n>0$.

Proof Here is the defining sentence of Vernikov [25, Theorem 3.1] formulated for $\mathbf{I}$ : the variety $\operatorname{var}\left\{x_{1} x_{2} \cdots x_{n} \approx 0\right\}$ is the largest nil-variety in $\mathbf{I}$ that contains $\mathcal{N}_{n}$ but does not contain $\mathcal{N}_{n+1}$.

According to [25, Proposition 6.2],

$$
\operatorname{var} \mathfrak{B}_{0,0, n}=\operatorname{var}\{x y \approx y x\} \vee \operatorname{var}\left\{x_{1} x_{2} \cdots x_{n} \approx 0\right\} .
$$

So, we have the following lemma.

Lemma 2.7 If I contains var $\mathfrak{B}_{0,0, n}$ for some $n>1$, then the variety var $\mathfrak{B}_{0,0, n}$ is definable in $\mathbf{I}$.

Lemma 2.7 generalizes [3, Corollary 8.2] that says that for each $n>1$ the variety $\operatorname{var} \mathfrak{B}_{0,0, n}$ is definable in $\mathbf{P}(\mathbf{0})$.

\section{Functions on varieties and sets of identities}

Definition 3.1 [17] If $\mathcal{V}$ is a variety of semigroups, then $m(\mathcal{V})$ and respectively $d(\mathcal{V})$ denote the maximal numbers $m$ and $d$ such that $\mathcal{V}$ contains the cyclic monoid (or group) $\mathbf{C}_{m, d}$.

In view of Lemmas 2.2 and 2.3, the functions $m=m(\mathcal{V})$ and $d=d(\mathcal{V})$ are definable in SEM.

If $x$ is a variable and $\tau$ is an identity, then $d(x, \tau)$ denotes the difference between the numbers of occurrences of $x$ in two sides of $\tau$. If $d(x, \tau)=0$, then variable $x$ is called balanced in $\tau$, otherwise $x$ is called unbalanced.

Let $m(\tau)$ denote the least number of times an unbalanced variable occurs in one side of $\tau$ and $d(\tau)$ denote the greatest common divisor of $d(x, \tau)$ among all variables $x$ in $\tau$. If $\tau$ is a balanced identity, then we set $m(\tau):=\infty$ and $d(\tau):=\infty$. 
Definition 3.2 [17] If $\Sigma$ is a set of identities, then $m(\Sigma)$ is the least number of times an unbalanced variable occurs in one side of an identity from $\Sigma$ and $d(\Sigma)$ is the greatest common divisor of all the differences between the numbers of occurrences of variables in two sides of identities from $\Sigma$. If all identities in $\Sigma$ are balanced, we set $m(\Sigma):=\infty$ and $d(\Sigma):=\infty$.

Lemma 3.1 ([17], Theorem 2) If $\Sigma$ is a set of identities, then $m(\operatorname{var} \Sigma)=m(\Sigma)$ and $d(\operatorname{var} \Sigma)=d(\Sigma)$.

The following lemma can be easily verified.

Lemma 3.2 If $\mathcal{V}$ and $\mathcal{U}$ are two semigroup varieties, then

(i) $m(\mathcal{V} \wedge \mathcal{U})=\min (m(\mathcal{V}), m(\mathcal{U}))$;

(ii) $d(\mathcal{V} \wedge \mathcal{U})=\operatorname{gcd}(d(\mathcal{V}), d(\mathcal{U}))$;

(iii) $m(\mathcal{V} \vee \mathcal{U})=\max (m(\mathcal{V}), m(\mathcal{U}))$;

(iv) $d(\mathcal{V} \vee \mathcal{U})=\operatorname{lcm}(d(\mathcal{V}), d(\mathcal{U}))$;

(v) if $\mathcal{V} \subseteq \mathcal{U}$, then $m(\mathcal{V}) \leq m(\mathcal{U})$ and $d(\mathcal{U})$ is a multiple of $d(\mathcal{V})$.

If $x$ is a variable and $u$ is a word, then $\operatorname{occ}_{u}(x)$ denotes the number of occurrences of $x$ in $u$. If $\operatorname{occ}_{u}(x)>0$, then we say that the word $u$ contains $x$. If $\operatorname{occ}_{u}(x)=1$, then we say that the variable $x$ is linear in $u$. The set of all variables contained in $u$ is called the content of $u$ (written $\operatorname{Cont}(u))$. Recall that an identity $u \approx v$ is called regular if $\operatorname{Cont}(u)=\operatorname{Cont}(v)$, otherwise it is called irregular. If $u$ is a word, then $|u|$ denotes the length of $u$ and $|\operatorname{Cont}(u)|$ denotes the number of distinct variables in $u$. Obviously, $|\operatorname{Cont}(u)| \leq|u|$ and $|\operatorname{Cont}(u)|=|u|$ if and only if each variable in $\operatorname{Cont}(u)$ is linear.

The next lemma will be used sometimes without a reference.

Lemma 3.3 Let $\Sigma$ and $\Delta$ be sets of identities such that $\operatorname{var} \Sigma=\operatorname{var} \Delta$. Then

(i) $m(\Sigma)=m(\Delta)$ and $d(\Sigma)=d(\Delta)$;

(ii) $\Sigma$ contains only regular identities if and only if so does $\Delta$;

(iii) $\Sigma$ contains only balanced identities if and only if so does $\Delta$.

Proof (i) Using Lemma 3.1, we have that $m(\Sigma)=m(\operatorname{var} \Sigma)=m(\operatorname{var} \Delta)=m(\Delta)$ and $d(\Sigma)=d(\operatorname{var} \Sigma)=d(\operatorname{var} \Delta)=d(\Delta)$.

(ii) It is clear that $\Sigma$ contains only regular identities if and only if $m(\Sigma)>0$. The rest follows from part (i).

(iii) Follows from part (i) and Definition 3.2.

Let $\Sigma$ be a set of balanced identities. If each identity in $\Sigma$ is trivial, we set $\ell(\Sigma)=$ $r(\Sigma)=\infty$. If $\Sigma$ contains a non-trivial identity, then $\ell(\Sigma)$ [respectively $r(\Sigma)$ ] is the least $\ell(\tau)$ [respectively $r(\tau)$ ] among all identities in $\Sigma$.

Lemma 3.4 If $\Sigma$ and $\Delta$ are sets of balanced identities such that $\operatorname{var} \Sigma=\operatorname{var} \Delta$, then $\ell(\Sigma)=\ell(\Delta)$ and $r(\Sigma)=r(\Delta)$. 
Proof If $\Sigma \vdash u \approx v$, then $\ell(u \approx v) \geq \ell=\ell(\Sigma)$ because the set $\Sigma$ does not contain any identities capable of changing the prefix of $u$ of length $\ell$. Similarly, $r(u \approx v) \geq$ $r(\Sigma)$.

Recall that a variety that satisfies an identity of the form $x^{t} \approx x^{t+d}$ is called periodic. The following proposition refines Proposition 1 in [17].

Proposition 3.1 For a semigroup variety $\mathcal{V}$ defined by a set of identities $\Sigma$, the following conditions are equivalent.

(i) $m(\mathcal{V})=m(\Sigma)<\infty$.

(ii) $d(\mathcal{V})=d(\Sigma)<\infty$.

(iii) $\Sigma$ contains an unbalanced identity.

(iv) $\mathcal{V}$ is a periodic variety.

(v) $\mathcal{V}$ satisfies an identity $x^{t} \approx x^{t+d}$ for some $t>0$ and $d=d(\mathcal{V})$.

Proof Conditions (i)-(iv) are equivalent by Proposition 1 in [17]. Since Condition (v) obviously implies Condition (iv), it is left to establish the implication (iv) $\rightarrow(\mathrm{v})$. Let $\mathcal{V}$ be a periodic variety and $k$ be the minimal number such that $\mathcal{V} \models x^{t} \approx x^{t+k}$ for some $t>0$. By the definition of the function $d(\mathcal{V})$ we have that $k$ is a multiple of $d=d(\mathcal{V})$. Suppose that $k>d$.

Claim $\mathcal{V} \models x^{p} \approx x^{p+c}$ for some $p>0$ and some $c>0$ such that $c$ is not a multiple of $k$.

Proof of Claim Since $d(\mathcal{V})=d$, the variety $\mathcal{V}$ satisfies some identity $\tau$ such that for some variable $x$ we have that $c=d(x, \tau)$ and $c$ is not a multiple of $k$. If $x$ is the only variable in $\tau$, then we are done.

Suppose now that the identity $\tau$ contains some variables other than $x$. Let $\gamma=$ $\gamma(x, y)$ be the identity obtained from $\tau$ by equalizing all variables other than $x$. If $r=d(y, \gamma)$ is a multiple of $k$, then the identities $x^{t} \approx x^{t+k}$ and $\gamma$ imply an identity of the form $x^{p} y^{q} \approx x^{p+c} y^{q}$ which in turn implies the desired identity.

Suppose now that $r=d(y, \gamma)$ is not a multiple of $k$. If $c+r$ (may be $c-r$ or $r-c$ depending on the situation) is not a multiple of $k$ then we can equalize $x$ and $y$ and obtain the desired identity.

If $c+r$ (or $c-r$ or $r-c$ ) is a multiple of $k$, then we substitute $y \rightarrow y y$ and denote the resulting identity by $\delta=\delta(x, y)$. Now we have that $d(x, \delta)=c, d(y, \delta)=2 r$ and $r$ is not a multiple of $k$. If we equalize $x$ and $y$ in $\delta$ then we obtain the desired identity. The claim is proved.

Now the identities $x^{t} \approx x^{t+k}$ and $x^{p} \approx x^{p+c}$ imply the identity $x^{t^{\prime}} \approx x^{t^{\prime}+k+c}$ for $t^{\prime}=\max (t, p)$. This identity together with the identity $x^{t} \approx x^{t+k}$ implies an identity $x^{t^{\prime}} \approx x^{t^{\prime}+k^{\prime}}$ for some $k^{\prime}<k$. This contradicts to the minimality of $k$. Therefore, $k=d$. 


\section{Definability of the set of all $b 0$-reduced varieties in I}

Recall that a nil-variety is called $b 0$-reduced if whenever it satisfies an unbalanced identity $u \approx v$, it also satisfies the identity $u \approx 0$ (and consequently, $v \approx 0$ ).

Lemma 4.1 If $\mathcal{V}$ is a b0-reduced variety, then for each prime $d>1$, the variety $\mathcal{V} \vee \operatorname{var} \mathbf{C}_{0, d}$ is a cover of the variety $\mathcal{V}$.

Proof Since $\mathcal{V}$ is a nil-variety, we have that $d(\mathcal{V})=1$. Since $d\left(\mathcal{V} \vee \operatorname{var} \mathbf{C}_{0, d}\right)=$ $\operatorname{lcm}(1, d)=d>1$, we have that $\mathcal{V} \subset \mathcal{V} \vee \operatorname{var} \mathbf{C}_{0, d}$. Let $\mathcal{V}^{\prime}$ be a variety such that $\mathcal{V} \subseteq \mathcal{V}^{\prime} \subseteq \mathcal{V} \vee \operatorname{var} \mathbf{C}_{0, d}$. Since the group $\mathbf{C}_{0, d}$ is commutative, the variety $\mathcal{V} \vee \operatorname{var} \mathbf{C}_{0, d}$ satisfies all balanced identities of $\mathcal{V}$. Therefore, the variety $\mathcal{V}^{\prime}$ also satisfies all balanced identities of $\mathcal{V}$.

If $d\left(\mathcal{V}^{\prime}\right)=d$, then by Definition 3.1, the variety $\mathcal{V}^{\prime}$ contains the cyclic group $\mathbf{C}_{0, d}$. Since $\mathcal{V} \subseteq \mathcal{V}^{\prime} \subseteq \mathcal{V} \vee \operatorname{var} \mathbf{C}_{0, d}$, the variety $\mathcal{V}^{\prime}$ coincides with $\mathcal{V} \vee \operatorname{var} \mathbf{C}_{0, d}$.

If $d\left(\mathcal{V}^{\prime}\right)=1$, then by Proposition 3.1 we have that $\mathcal{V}^{\prime} \models x^{p} \approx x^{p+1}$ for some $p>0$. Let $a \approx 0$ be some 0 -reduced identity satisfied by $\mathcal{V}$. Then $\mathcal{V} \vee \operatorname{var} \mathbf{C}_{0, d}$ satisfies $a \approx a x^{d} \approx x^{d} a$ for some variable $x$ that does not occur in the word $a$. Since $\mathcal{V}^{\prime} \subseteq \mathcal{V} \vee \operatorname{var} \mathbf{C}_{0, d}$, the variety $\mathcal{V}^{\prime}$ also satisfies $a \approx a x^{d} \approx x^{d} a$. Now the identities $a \approx a x^{d} \approx x^{d} a$ imply $a \approx a x^{k d} \approx x^{k d} a$ for some $k>0$ such that $k d \geq p$. The latter identities together with $x^{p} \approx x^{p+1}$ imply $a \approx a x^{k d} \approx a x^{k d+1} \approx a x$ and similarly, $a \approx x a$. Therefore, $\mathcal{V}^{\prime} \models a \approx 0$. Since the variety $\mathcal{V}$ is $b 0$-reduced and $\mathcal{V}^{\prime}$ satisfies all balanced and all 0 -reduced identities of $\mathcal{V}$, the variety $\mathcal{V}^{\prime}$ coincides with $\mathcal{V}$. Therefore, the variety $\mathcal{V} \vee \operatorname{var} \mathbf{C}_{0, d}$ is a cover of the variety $\mathcal{V}$.

If $\mathcal{V}$ is a variety then $\operatorname{Nil}(\mathcal{V})$ denotes the set of all nil-semigroups contained in $\mathcal{V}$. If the variety $\mathcal{V}$ satisfies an identity of the form $x^{t} \approx x^{t+d}$, then it is easy to verify that $\operatorname{Nil}(\mathcal{V})$ is a subvariety of $\mathcal{V}$ that can be defined within $\mathcal{V}$ by the identity $x^{t} \approx 0$. In this case, $\operatorname{Nil}(\mathcal{V})$ is, obviously, the greatest nil-subvariety of $\mathcal{V}$.

The next lemma generalizes Lemmas 1 and 8 in [28] and is proved in [23] similarly to [28, Lemma 8].

Lemma 4.2 ([23], Lemma 2.8) Let $\mathcal{V}$ be a nil-variety that satisfies $u \approx v$ and does not satisfy $u \approx 0$. If $\mathcal{U}$ is a variety that does not satisfy $u \approx v$, then $\mathcal{V}$ is a proper subset of $\operatorname{Nil}(\mathcal{V} \vee \mathcal{U})$.

Corollary 4.1 If a nil-variety $\mathcal{V}$ is not b0-reduced, then for some prime $d$, the variety $\mathcal{V} \vee \operatorname{var} \mathbf{C}_{0, d}$ is not a cover of the variety $\mathcal{V}$.

Proof If the variety $\mathcal{V}$ is not $b 0$-reduced, then $\mathcal{V}$ satisfies some unbalanced identity $u \approx v$ and does not satisfy the identity $u \approx 0$. If $d>d(u \approx v)$, then $\operatorname{var} \mathbf{C}_{0, d}$ does not satisfy the identity $u \approx v$. Since the variety $\mathcal{V} \vee \operatorname{var} \mathbf{C}_{0, d}$ is periodic, the set $\mathcal{N}=$ $\operatorname{Nil}\left(\mathcal{V} \vee \operatorname{var} \mathbf{C}_{0, d}\right)$ is a subvariety of $\mathcal{V} \vee \operatorname{var} \mathbf{C}_{0, d}$. By Lemma 4.2, the variety $\mathcal{V}$ is a proper subvariety of $\mathcal{N}$. Now $\mathcal{N}$, in turn, is a proper subvariety of $\mathcal{V} \vee \operatorname{var} \mathbf{C}_{0, d}$ because for $d>1$ the cyclic group $\mathbf{C}_{0, d}$ is not a nil-semigroup. Therefore, the variety $\mathcal{V} \vee \operatorname{var} \mathbf{C}_{0, d}$ is not a cover of $\mathcal{V}$. 
Lemma 4.1 and Corollary 4.1 immediately imply the following.

Proposition 4.1 A nil-variety $\mathcal{V}$ is b0-reduced if and only if for each prime $d>1$, the variety $\mathcal{V} \vee \operatorname{var} \mathbf{C}_{0, d}$ is a cover of the variety $\mathcal{V}$.

Proposition 4.1 and Lemmas 2.1 and 2.4 imply the following.

Theorem 4.1 Let $\mathbf{I}$ be a sublattice of SEM that contains $\operatorname{var}\{x y \approx y x\}$ and also contains $L(\mathcal{V})$ whenever $\mathcal{V} \in \mathbf{I}$. Then the set of all b0-reduced varieties in $\mathbf{I}$ is definable in $\mathbf{I}$. In particular, the set of all b0-reduced varieties is definable in SEM and the set of all commutative b0-reduced varieties is definable in COM.

\section{A quasi-order on the free semigroup modulo $\mathfrak{B}$}

By $F_{\infty}$ we denote the free semigroup over a countably infinite alphabet, i.e. the semigroup of words under concatenation. If $\mathfrak{B}$ is a set of regular identities and $v$ and $u$ are words, then we define $v \leq_{\mathfrak{B}} u$ if and only if $\operatorname{var}\{v \approx 0, \mathfrak{B}\} \models u \approx 0$. If the set $\mathfrak{B}$ contains only trivial identities, then instead of $\leq_{\mathfrak{B}}$ we simply write $\leq$. The relation $\leq$ on the free semigroup is well-known and can be defined as follows: if $u, v \in F_{\infty}$, then $v \leq u$ if and only if $u=a \Theta(v) b$ for some possibly empty words $a$ and $b$ and some substitution $\Theta$.

It is easy to see that the relation $\leq_{\mathfrak{B}}$ is reflexive and transitive, i.e. it is a quasiorder on the free semigroup $F_{\infty}$. If $u \leq_{\mathfrak{B}} v \leq_{\mathfrak{B}} u$, then we write $u \Leftrightarrow_{\mathfrak{B}} v$. If $u$ is a word, then the class of all words equivalent to $u$ modulo $\Leftrightarrow_{\mathfrak{B}}$ is denoted by $[u]_{\Leftrightarrow \mathfrak{B}}$. Let $F_{\infty} / \Leftrightarrow_{\mathfrak{B}}$ denote the set of all classes $[u]_{\Leftrightarrow_{\mathfrak{B}}}$ ordered by $\leq_{\mathfrak{B}}$. Following [11], the elements of the ordered set $F_{\infty} / \Leftrightarrow_{\mathfrak{B}}$ will be called word patterns modulo $\mathfrak{B}$.

It is easy to see that each substitution (i.e. an endomorphism of the free semigroup) is a composition of the following three types of elementary substitutions:

1. One-to-one renaming of variables. (Such a substitution will be simply called renaming of variables.)

2. Equalizing two variables $x$ and $y$ (renaming $y$ by $x$ ).

3. Substitution of the form $x \rightarrow x y(x \rightarrow y x)$.

Lemma 5.1 If $u \leq v$, then $|u|-|\operatorname{Cont}(u)| \leq|v|-|\operatorname{Cont}(v)|$.

Proof This statement can be easily verified in the following two cases.

Case 1: $v=u x$ or $v=x u$ for some new variable $x$.

Case 2: $v=\Theta(u)$ where $\Theta$ is an elementary substitution.

The general case follows by transitivity.

If $\mathfrak{B}$ is a set of identities, then $\sim_{\mathfrak{B}}$ denotes the fully invariant congruence on the free semigroup corresponding to $\mathfrak{B}$. The next two lemmas establish the connections between the quasi-orders $\leq_{\mathfrak{B}}$ and $\leq$. 
Lemma 5.2 If $\mathfrak{B}$ is a set of regular identities, then the following are equivalent:

(i) $v \leq_{\mathfrak{B}} u$;

(ii) there exists a word $u^{\prime}$ such that $u \sim_{\mathfrak{B}} u^{\prime}$ and $v \leq u^{\prime}$.

Proof (i) $\rightarrow$ (ii). If $v \leq_{\mathfrak{B}} u$, then there exists a sequence of words $u_{1}, \ldots, u_{n}$ such that one can derive $u \approx 0$ from $\{v \approx 0, \mathfrak{B}\}$ as follows: $u=u_{1} \approx u_{2} \approx \ldots \approx u_{n} \approx 0$. Since $m(\mathfrak{B})>0$ but $m(u \approx 0)=0$, we cannot derive $u \approx 0$ from $\mathfrak{B}$ without using $v \approx 0$. Once the identity $v \approx 0$ is used, the derivation of $u \approx 0$ is finished. So, $u \sim_{\mathfrak{B}}$ $u_{n}$ and $v \leq u_{n}$.

(ii) $\rightarrow$ (i). Evident.

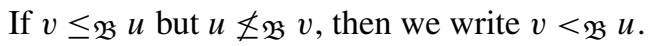

Lemma 5.3 If $\mathfrak{B}$ is a set of balanced identities, then:

(i) $u \Leftrightarrow \mathfrak{B} v$ if and only if $u \sim_{\mathfrak{B}} p(v)$ for some renaming $p$ of variables;

(ii) if $u \Leftrightarrow \mathfrak{B} v$, then $|u|=|v|$;

(iii) if $v \leq_{\mathfrak{B}} u$ and $|v|<|u|$, then $v<_{\mathfrak{B}} u$;

(iv) $v<_{\mathfrak{B}} u$ if and only if there exists a word $u^{\prime}$ such that $u \sim_{\mathfrak{B}} u^{\prime}$ and $v<u^{\prime}$.

Proof (i) If $v \sim_{\mathfrak{B}} p(u)$, then $u \approx p(v) \approx 0$ is a derivation of $u \approx 0$ from $\{v \approx 0, \mathfrak{B}\}$. The identity $v \approx 0$ can be derived from $\{u \approx 0, \mathfrak{B}\}$ in a similar way. So, $u \Leftrightarrow_{\mathfrak{B}} v$.

If $v \Leftrightarrow \mathfrak{B} u$, then by Lemma 5.2, one can find words $u^{\prime}$ and $v^{\prime}$ such that $u \sim_{\mathfrak{B}} u^{\prime}$, $v \leq u^{\prime}$ and $v \sim_{\mathfrak{B}} v^{\prime}, u \leq v^{\prime}$. Since the identities $u \approx u^{\prime}$ and $v \approx v^{\prime}$ are balanced, all the words $u, u^{\prime}, v$ and $v^{\prime}$ have the same length. Therefore, $u^{\prime}=p(v)$ for some substitution $p$ that maps variables to variables. Suppose that for some distinct variables $x$ and $y$ with $\operatorname{occ}_{v}(x)>0$ and $\operatorname{occ}_{v}(y)>0$ we have that $p(x)=p(y)=z$. But then $|u|-|\operatorname{Cont}(u)|=\left|u^{\prime}\right|-\left|\operatorname{Cont}\left(u^{\prime}\right)\right|>|v|-|\operatorname{Cont}(v)|=\left|v^{\prime}\right|-\left|\operatorname{Cont}\left(v^{\prime}\right)\right|$. In view of Lemma 5.1, this contradicts the fact that $u \leq v^{\prime}$. Therefore, the substitution $p$ is a renaming of variables.

(ii) If $u \Leftrightarrow \mathfrak{B} v$, then by part (i) the identity $u \approx p(v)$ is balanced. Therefore, $|u|=$ $|p(v)|=|v|$.

(iii) If $u \Leftrightarrow_{\mathfrak{B}} v$, then by part (ii) we have that $|u|=|v|$. Therefore $v<_{\mathfrak{B}} u$.

(iv) Since $v<_{\mathfrak{B}} u$, by Lemma 5.2 one can find a word $u^{\prime}$ such that $u \sim_{\mathfrak{B}} u^{\prime}$ and $v \leq u^{\prime}$. So, $u^{\prime}=a \Theta(v) b$ for some possibly empty word $a b$ and a substitution $\Theta$. If the word $a b$ is empty and $\Theta$ is a renaming of variables, then by part (i) we have that $u \Leftrightarrow_{\mathfrak{B}} v$. Since the words $v$ and $u$ are not equivalent modulo $\Leftrightarrow_{\mathfrak{B}}$, we have that $v<u^{\prime}$.

Now suppose that there is a word $u^{\prime}$ such that $u \sim_{\mathfrak{B}} u^{\prime}$ and $v<u^{\prime}$. Then by Lemma 5.2 we have that $v \leq_{\mathfrak{B}} u$. If $|v|<|u|$ then by part (iii) we have that $v<_{\mathfrak{B}} u$.

So, we may assume that $|u|=|v|$ and consequently $|u|=|v|=\left|u^{\prime}\right|$. Since $v<u^{\prime}$ and $|v|=\left|u^{\prime}\right|$ we have that $u^{\prime}=\Theta(v)$ for some substitution $\Theta$ that maps variables to variables such that $\Theta$ is not a renaming of variables. So, $u \sim_{\mathfrak{B}} \Theta(v)$. If we assume that $u \Leftrightarrow_{\mathfrak{B}} v$ then by part (i) we would have that $u \sim_{\mathfrak{B}} p(v)$ for some renaming of variables $p$. Thus the identity $\Theta(v) \approx p(v)$ would be balanced which is not the case. Therefore, $v<\mathfrak{B} u$. 
The following lemma is needed only to justify Theorem 6.1 and sometimes will be used without a reference.

Lemma 5.4 If $\mathfrak{B}$ is a set of balanced identities with $\ell(\mathfrak{B})>0$, then:

(i) the words $x^{2} y_{1} \cdots y_{k}$ and $y_{1} \cdots y_{k} x^{2}$ are not equivalent modulo $\Leftrightarrow_{\mathcal{B}}$ for any $k>0$;

(ii) the words $x^{k} y$ and $y x^{k}$ are not equivalent modulo $\Leftrightarrow_{\mathcal{B}}$ for any $k>0$;

(iii) the words $y_{1} \cdots y_{j} x^{k+2} t_{1} \cdots t_{p}$ and $y_{1} \cdots y_{j+1} x^{k+1} t_{1} \cdots t_{p}$ are not equivalent modulo $\Leftrightarrow \mathcal{B}$ for any $k \geq 0$.

Proof (i) If $x^{2} y_{1} \cdots y_{k} \Leftrightarrow \mathcal{B} y_{1} \cdots y_{k} x^{2}$, then Lemma 5.3(i) implies $x^{2} y_{1} \cdots y_{k} \sim_{\mathfrak{B}}$ $p\left(y_{1} \cdots y_{k} x^{2}\right)$ for some renaming of variables $p$. Since $x$ is the only non-linear variable in both words, this can only happen if $\mathfrak{B} \vdash x^{2} y_{1} \cdots y_{k} \approx y_{1} \cdots y_{k} x^{2}$ which is impossible in view of Lemma 3.4.

(ii) Similar to the proof of (i).

(iii) If $y_{1} \cdots y_{j} x^{k+2} t_{1} \cdots t_{p} \Leftrightarrow \mathcal{B} y_{1} \cdots y_{j+1} x^{k+1} t_{1} \cdots t_{p}$ then by Lemma 5.3(i) we have that $y_{1} \cdots y_{j} x^{k+2} t_{1} \cdots t_{p} \sim_{\mathfrak{B}} q\left(y_{1} \cdots y_{j+1} x^{k+1} t_{1} \cdots t_{p}\right)$ for some renaming of variables $q$. Since $x$ is the only non-linear variable in both words and occurs different number of times in each of the word, this is impossible in view of Lemma 3.3(iii).

\section{Definability of each word pattern modulo $\mathfrak{B}$ in the ordered set of all such patterns}

\section{Lemma 6.1 [12, Theorem 4.3]}

(i) For each word $u$ the pattern $[u]_{\Leftrightarrow_{x y \approx y x}}$ is definable in the ordered set $F_{\infty} /$ $\Leftrightarrow{ }_{x y \approx y x}$ of all such patterns.

(ii) $F_{\infty} / \Leftrightarrow_{\mathfrak{B}_{0,0,3}}=F_{\infty} / \Leftrightarrow_{x y \approx y x}$.

(iii) Each word pattern $[u]_{\Leftrightarrow_{\mathfrak{B}_{0,0,3}}}$ is definable in the ordered set $F_{\infty} / \Leftrightarrow_{\mathfrak{B}_{0,0,3}}$.

Proof (i) can be justified by a simple argument of McKenzie contained in the proof of Theorem 4.3 in [12].

(ii) If $|u|>2$, then $[u]_{\sim_{x y \approx y x}}=[u]_{\mathcal{B}_{0,0,3}}$. Therefore, by Lemma 5.3 we have that $[u]_{\Leftrightarrow x y \approx y x}=[u]_{\Leftrightarrow \mathcal{B}_{0,0,3}}$.

All equivalence classes $[u]=[u]_{\Leftrightarrow_{\mathfrak{B}_{0,0,3}}}=[u]_{\Leftrightarrow_{x y \approx y x}}$ for words $u$ with $|u|<3$ are the following: $\left\{[x y],\left[x^{2}\right],[x]\right\}$.

(iii) follows from parts (i) and (ii).

Obviously, if var $\mathfrak{B} \subset$ var $\mathfrak{C}$, then $v \leq_{\mathfrak{C}} u$ implies $v \leq_{\mathfrak{B}} u$. In particular, $v \leq u$ implies $v \leq_{\mathfrak{B}} u$ for every $\mathfrak{B}$. If $\mathfrak{B}$ contains only balanced identities, then $v \leq_{\mathfrak{B}} u$ implies $v \leq_{x y \approx y x} u$, and, consequently, $u \Leftrightarrow_{\mathfrak{B}} v \Rightarrow u \Leftrightarrow_{x y \approx y x} v$. Therefore, for each word $u$ the class $[u]_{\Leftrightarrow_{x y \approx y x}}$ is a union of equivalence classes modulo $\Leftrightarrow_{\mathfrak{B}}$. If $\mathfrak{B}$ is set of balanced identities and $u$ is a word, then $S_{u, \mathfrak{B}}$ denotes the set of all classes $[v]_{\Leftrightarrow_{\mathfrak{B}}}$ contained in the class $[u]_{\Leftrightarrow y \approx y x}$. 
Lemma 6.2 If $\mathfrak{B}$ is a set of balanced identities, then for each word $u$ the set $S_{u, \mathfrak{B}}$ is definable in $F_{\infty} / \Leftrightarrow_{\mathfrak{B}}$.

Proof By Lemma 6.1, for each word $u$ there is a first-order formula that defines the pattern $[u]_{\Leftrightarrow x \approx \approx y x}$ in the ordered set $F_{\infty} / \Leftrightarrow_{x y \approx y x}$. Since for every two words $v$ and $u$ we have that $v \leq_{\mathfrak{B}} u \Longrightarrow v \leq_{x y \approx y x} u$, the same formula defines the set $S_{u, \mathfrak{B}}$ in $F_{\infty} / \Leftrightarrow_{\mathfrak{B}}$.

If $\mathfrak{B}$ is a set of regular identities, then the set $F_{\infty} / \Leftrightarrow_{\mathfrak{B}}$ ordered by $\leq_{\mathfrak{B}}$ can be identified with the set of all varieties of the form $\operatorname{var}\{u \approx 0, \mathfrak{B}\}$ ordered under inclusion.

Lemma 6.3 For a set of balanced identities $\mathfrak{B}$, the following sets of patterns are definable in the ordered set $F_{\infty} / \Leftrightarrow_{\mathfrak{B}}$ :

(i) $\left\{\left[x^{k}\right]_{\Leftrightarrow_{\mathfrak{B}}}\right\}$ and $\left\{\left[x_{1} \cdots x_{k}\right]_{\Leftrightarrow_{\mathfrak{B}}}\right\}$ for each $k>0$;

(ii) $\left\{\left[x^{2} y\right]_{\Leftrightarrow \mathfrak{B}},\left[y x^{2}\right]_{\Leftrightarrow \mathfrak{B}}\right\}$.

Proof Part (i) follows from Lemma 6.2 and the fact that for each $k>0$, the sets $S_{x^{k}, \mathfrak{B}}$ and $S_{x_{1} \cdots x_{k}, \mathfrak{B}}$ are singletons.

(ii) The set $S_{x^{2} y, \mathfrak{B}}$ contains at most three varieties, namely $\operatorname{var}\left\{x^{2} y \approx 0, \mathfrak{B}\right\}$, $\operatorname{var}\left\{y x^{2} \approx 0, \mathfrak{B}\right\}$ and $\operatorname{var}\{x y x \approx 0, \mathfrak{B}\}$. If the variety $\operatorname{var}\{x y x \approx 0, \mathfrak{B}\}$ is different from $\operatorname{var}\left\{y x^{2} \approx 0, \mathfrak{B}\right\}$ and $\operatorname{var}\left\{x^{2} y \approx 0, \mathfrak{B}\right\}$, then it can be told apart from the other two by saying that it does not contain $\operatorname{var}\left\{x^{2} \approx 0, \mathfrak{B}\right\}$.

Lemma 6.4 ([3], Theorem 9.3) For every $n>3$ and each word $u$, the pattern $[u]_{\Leftrightarrow_{\mathfrak{B}_{0,0, n}}}$ is definable in the ordered set $F_{\infty} / \Leftrightarrow_{\mathfrak{B}_{0,0, n}}$ assuming that the pattern $\left[x^{2} y\right]_{\mathfrak{B}_{0,0, n}}$ is definable in $F_{\infty} / \Leftrightarrow_{\mathfrak{B}_{0,0, n}}$.

The next theorem generalizes [11, Proposition 2.8] and we prove it by repeating the arguments used to prove Theorem 9.3 in [3].

Theorem 6.1 If $\mathfrak{B}$ is a set of balanced identities with $\ell(\mathfrak{B})>0$ or $r(\mathfrak{B})>0$, then each word pattern modulo $\mathfrak{B}$ is definable in the ordered set $F_{\infty} / \Leftrightarrow_{\mathfrak{B}}$ assuming that the pattern $\left[x^{2} y\right]_{\Leftrightarrow_{\mathfrak{B}}}$ is definable in $F_{\infty} / \Leftrightarrow_{\mathfrak{B}}$.

Proof Without loss of generality, we may assume that $\ell=\ell(\mathfrak{B})>0$. We regard word patterns as varieties of the form $\operatorname{var}\{u \approx 0, \mathfrak{B}\}$ ordered under inclusion. In view of Lemma 6.2, we only need to show how to distinguish between the varieties in the set $S_{u, \mathfrak{B}}$ for each word $u$.

Claim 1 For each $k \geq 0$, the varieties

$$
\operatorname{var}\left\{x^{2} y_{1} \cdots y_{k} \approx 0, \mathfrak{B}\right\} \text { and } \operatorname{var}\left\{y_{1} \cdots y_{k} x^{2} \approx 0, \mathfrak{B}\right\}
$$

are definable in $F_{\infty} / \Leftrightarrow_{\mathfrak{B}}$ assuming that $\operatorname{var}\left\{x^{2} y \approx 0, \mathfrak{B}\right\}$ is definable in $F_{\infty} / \Leftrightarrow_{\mathfrak{B}}$. 
Proof of Claim 1 In view of Lemma 6.3, we may assume that $k>1$. Since $\ell>0$, the varieties $\operatorname{var}\left\{x^{2} y_{1} \cdots y_{k} \approx 0, \mathfrak{B}\right\}$ and $\operatorname{var}\left\{y_{1} \cdots y_{k} x^{2} \approx 0, \mathfrak{B}\right\}$ are different from each other. As in [3], we tell apart the variety $\operatorname{var}\left\{x^{2} y_{1} \cdots y_{k} \approx 0, \mathfrak{B}\right\}$ from all other varieties in $S_{x^{2} y_{1} \cdots y_{k}, \mathfrak{B}}$ by saying that it is a cover of the variety $\operatorname{var}\left\{x^{2} y_{1} \cdots y_{k-1} \approx 0, \mathfrak{B}\right\}$ but does not contain $\operatorname{var}\left\{y x^{2} \approx 0, \mathfrak{B}\right\}$. Dually, we tell apart the variety $\operatorname{var}\left\{y_{1} \cdots y_{k} x^{2} \approx 0, \mathfrak{B}\right\}$ from all other varieties in $S_{x^{2} y_{1} \cdots y_{k}, \mathfrak{B}}$ by saying that it is a cover of $\operatorname{var}\left\{y_{1} \cdots y_{k-1} x^{2} \approx 0, \mathfrak{B}\right\}$ but does not contain $\operatorname{var}\left\{x^{2} y \approx 0, \mathfrak{B}\right\}$. So, by induction, the varieties $\operatorname{var}\left\{x^{2} y_{1} \cdots y_{k} \approx 0, \mathfrak{B}\right\}$ and $\operatorname{var}\left\{y_{1} \cdots y_{k} x^{2} \approx 0, \mathfrak{B}\right\}$ are definable in $F_{\infty} / \Leftrightarrow_{\mathfrak{B}}$. Claim 1 is proved.

Claim 2 For each $k>0$, the varieties $\operatorname{var}\left\{x^{k} y \approx 0, \mathfrak{B}\right\}$ and $\operatorname{var}\left\{y x^{k} \approx 0, \mathfrak{B}\right\}$ are definable in $F_{\infty} / \Leftrightarrow_{\mathfrak{B}}$ assuming that $\operatorname{var}\left\{x^{2} y \approx 0, \mathfrak{B}\right\}$ is definable in $F_{\infty} / \Leftrightarrow_{\mathfrak{B}}$.

Proof of Claim 2 In view of Lemma 6.3, we may assume that $k \geq 3$. As in [3], we tell apart the variety $\operatorname{var}\left\{x^{k} y \approx 0, \mathfrak{B}\right\}$ from all other varieties in $S_{x^{k} y, \mathfrak{B}}$ by saying that it contains $\operatorname{var}\left\{x^{2} y_{1} \cdots y_{k-1} \approx 0, \mathfrak{B}\right\}$ and $\operatorname{var}\left\{x^{k} \approx 0, \mathfrak{B}\right\}$ but does not contain $\operatorname{var}\left\{y_{1} \cdots y_{k-1} x^{2} \approx 0, \mathfrak{B}\right\}$. Claim 2 is proved by induction and duality.

Claim 3 For each $k+p>0$, the varieties $\operatorname{var}\left\{x^{k} y_{1} \cdots y_{p} \approx 0, \mathfrak{B}\right\}$ and $\operatorname{var}\left\{y_{1} \cdots y_{p} x^{k} \approx 0, \mathfrak{B}\right\}$ are definable in $F_{\infty} / \Leftrightarrow_{\mathfrak{B}}$ assuming that $\operatorname{var}\left\{x^{2} y \approx 0, \mathfrak{B}\right\}$ is definable in $F_{\infty} / \Leftrightarrow_{\mathfrak{B}}$.

Proof of Claim 3 In view of the previous claims, we assume that $k \geq 3$ and $p \geq 2$. As in [3], we tell apart the variety $\operatorname{var}\left\{x^{k} y_{1} \cdots y_{p} \approx 0, \mathfrak{B}\right\}$ from all other varieties in $S_{x^{k} y_{1} \cdots y_{p}, \mathfrak{B}}$ by saying that it contains $\operatorname{var}\left\{x^{k} y_{1} \cdots y_{p-1} \approx 0, \mathfrak{B}\right\}$ but does not contain $\operatorname{var}\left\{y x^{k} \approx 0, \mathfrak{B}\right\}$. Claim 3 is proved by induction and duality.

Claim 4 For each $k+p+j>0$, the variety $\operatorname{var}\left\{y_{1} \cdots y_{p} x^{k} z_{1} \cdots z_{j} \approx 0, \mathfrak{B}\right\}$ is definable in $F_{\infty} / \Leftrightarrow_{\mathfrak{B}}$ assuming that $\operatorname{var}\left\{x^{2} y \approx 0, \mathfrak{B}\right\}$ is definable in $F_{\infty} / \Leftrightarrow_{\mathfrak{B}}$.

Proof of Claim 4 In view of the previous claims, we assume that $k \geq 3, p>1$ and $j>1$. As in [3], we tell apart the variety $\operatorname{var}\left\{y_{1} \cdots y_{p} x^{k} z_{1} \cdots z_{j} \approx 0, \mathfrak{B}\right\}$ from all other varieties in $S_{y_{1} \cdots y_{p} x^{k} z_{1} \cdots z_{j}, \mathfrak{B}}$ by saying that it contains both $\operatorname{var}\left\{y_{1} \cdots y_{p} x^{k} \approx 0, \mathfrak{B}\right\}$ and $\operatorname{var}\left\{x^{k} z_{1} \cdots z_{j} \approx 0, \mathfrak{B}\right\}$. Claim 4 is proved by induction.

Claim 5 For each $k>0$, the variety

$$
\mathcal{V}_{j, k, p}=\operatorname{var}\left\{y_{1} \cdots y_{j} x z_{1} \cdots z_{k} x t_{1} \cdots t_{p} \approx 0, \mathfrak{B}\right\}
$$

is definable in $F_{\infty} / \Leftrightarrow_{\mathfrak{B}}$ assuming that $\operatorname{var}\left\{x^{2} y \approx 0, \mathfrak{B}\right\}$ is definable in $F_{\infty} / \Leftrightarrow_{\mathfrak{B}}$.

Proof of Claim 5 As in [3], we tell apart the variety $\mathcal{V}_{j, k, p}$ from all other varieties in $S_{y_{1} \cdots y_{j} x z_{1} \cdots z_{k} x t_{1} \cdots t_{p}, \mathfrak{B}}$ by saying that $\mathcal{V}_{j, k, p}$ is contained in the variety 
$\operatorname{var}\left\{y_{1} \cdots y_{j} x^{k+2} t_{1} \cdots t_{p} \approx 0, \mathfrak{B}\right\}$ but in neither $\operatorname{var}\left\{y_{1} \cdots y_{j+1} x^{k+1} t_{1} \cdots t_{p} \approx 0, \mathfrak{B}\right\}$ nor $\operatorname{var}\left\{y_{1} \cdots y_{j} x^{k+1} t_{1} \cdots t_{p+1} \approx 0, \mathfrak{B}\right\}$. Claim 5 is proved by induction.

Now let $u=x_{1} \cdots x_{k}$ be an arbitrary non-linear word. The final argument in [3] is that we can tell apart the variety $\operatorname{var}\{u \approx 0, \mathfrak{B}\}$ from all other varieties in $S_{u, \mathfrak{B}}$ by saying that for each $i, j(1 \leq i<j \leq k)$ such that $x_{i}=x_{j}$ the variety $\operatorname{var}\{u \approx 0, \mathfrak{B}\}$ contains the variety $\mathcal{V}_{j, k, p}$.

If $u$ is a word, then $u^{\delta}$ denotes the dual of $u$, i.e. the word $u$ written backward. The map $\delta: u \mapsto u^{\delta}$ induces the duality map on sets of identities. We say that a set of identities $\Sigma$ is self-dual if $\Sigma=\Sigma^{\delta}$. For example, for each $n>1$ the set $\mathfrak{B}_{\ell, r, n}$ is self-dual if and only if $\ell=r$.

If $\mathfrak{B}$ is a self-dual set of regular identities, then evidently, $v \leq_{\mathfrak{B}} u$ if and only if $v^{\delta} \leq_{\mathfrak{B}} u^{\delta}$. In this case, the map $[u]_{\Leftrightarrow_{\mathfrak{B}}} \mapsto\left[u^{\delta}\right]_{\Leftrightarrow_{\mathfrak{B}}}$ is well-defined and is an automorphism of $F_{\infty} / \Leftrightarrow_{\mathfrak{B}}$.

Extending the definition from [11] to arbitrary self-dual sets $\mathfrak{B}$ of regular identities, we say that a pattern $[u]_{\Leftrightarrow_{\mathfrak{B}}}$ is semi-definable in $F_{\infty} / \Leftrightarrow_{\mathfrak{B}}$ if one can find a first-order formula $\Phi_{u}(\bar{x}, \bar{y})$ that turns into a true statement on $F_{\infty} / \Leftrightarrow_{\mathfrak{B}}$ if and only if $\bar{x}=[u]_{\Leftrightarrow_{\mathfrak{B}}}$ and $\bar{y}=\left[x^{2} y\right]_{\Leftrightarrow_{\mathfrak{B}}}$, or else $\bar{x}=\left[u^{\delta}\right]_{\Leftrightarrow_{\mathfrak{B}}}$ and $\bar{y}=\left[y x^{2}\right]_{\Leftrightarrow_{\mathfrak{B}}}$.

Corollary 6.1 Let $\mathfrak{B}$ be a self-dual set of balanced identities with $\ell(\mathfrak{B})=r(\mathfrak{B})>0$ or $\mathfrak{B}=\mathfrak{B}_{k, k, n}$ for some $k \geq 0$ and $n>1$. Then each word pattern is semi-definable in the ordered set $F_{\infty} / \Leftrightarrow_{\mathfrak{B}}$.

Proof For every word $u$ the arguments used to prove Lemma 6.4 and Theorem 6.1 can be easily translated into a first-order formula $\Phi_{u}(\bar{x}, \bar{y})$ that turns into a true statement if and only if $\bar{x}=[u]_{\Leftrightarrow_{\mathfrak{B}}}$ and $\bar{y}=\left[x^{2} y\right]_{\Leftrightarrow_{\mathfrak{B}}}$, or else $\bar{x}=\left[u^{\delta}\right]_{\Leftrightarrow_{\mathfrak{B}}}$ and $\bar{y}=\left[y x^{2}\right]_{\Leftrightarrow_{\mathfrak{B}}}$.

For example, $\Phi_{x^{2} y_{1} y_{2}}(\bar{x}, \bar{y})$ is the following formula:

$$
\operatorname{Cov}(\bar{x}, \bar{y}) \& \Phi_{x^{2} y}(\bar{y}) \& \forall \bar{z}\left(\Phi_{x^{2} y}(\bar{z}) \& \bar{z} \neq \bar{y} \longrightarrow \bar{x} \nsucceq \bar{z}\right) .
$$

Here $\operatorname{Cov}(\bar{x}, \bar{y})$ is the formula that says that $\bar{x}$ is a cover of $\bar{y}$ and $\Phi_{x^{2} y}(\bar{x})$ is the formula in Lemma 6.3 that defines the set $\left\{\left[x^{2} y\right]_{\Leftrightarrow_{\mathfrak{B}}},\left[y x^{2}\right]_{\Leftrightarrow_{\mathfrak{B}}}\right\}$.

By induction, $\Phi_{x^{2} y_{1} y_{2} y_{3}}(\bar{x}, \bar{y})$ is the following formula:

$$
(\forall \bar{p}, z) \quad\left(\left(\Phi_{x^{2} y_{1} y_{2}}(\bar{p}, \bar{y}) \& \Phi_{x^{2} y_{1} y_{2}}(\bar{z}, \bar{y}) \& \bar{z} \neq \bar{p}\right) \longrightarrow(\operatorname{Cov}(\bar{x}, \bar{p}) \& \bar{x} \nsucceq \bar{z})\right) .
$$

The defining sentences used in Claims 2-5 can be translated into first-order formulas with two free variables in a similar way.

Corollary 6.2 For each $\ell \neq r \geq 0$ and $n>1$ each word pattern is definable in the ordered set $F_{\infty} / \Leftrightarrow_{\mathfrak{B}_{\ell, r, n}}$.

Proof For each word $u$ we denote the pattern $[u]_{\Leftrightarrow \mathfrak{B}_{\ell, r, n}}$ simply by $[u]$. Without loss of generality, we may assume that $\ell<r$. In view of Theorem 6.1 we only need to prove that the word pattern $\left[y x^{2}\right]$ is definable in $F_{\infty} / \Leftrightarrow_{\mathfrak{B}_{\ell, r, n}}$. 
To simplify the notation, we define some abbreviations for the elements $[u] \in$ $S_{m}=S_{x^{2} y_{1} y_{2} \cdots y_{m}, \mathfrak{B}_{\ell, r, n}}$ when $m \geq \ell+n+r-2$. If there are $i<\ell$ letters before the first occurrence of $x$ in $u$ and $j<\ell$ letters before the second occurrence of $x$, then we denote $[u]=L_{i} L_{j}$. If there are $i<\ell$ letters before the first occurrence of $x$ in $u$, at least $\ell$ letters before and at least $r$ letters after the second occurrence of $x$, then we denote $[u]=L_{i} M$. If there are $i<\ell$ letters before the first occurrence of $x$ in $u$ and $j<r$ letters after the second occurrence of $x$, then we denote $[u]=L_{i} R_{j}$. If there are at least $\ell$ letters before the first occurrence of $x$ in $u$ and at least $r$ letters after the second occurrence of $x$, then we denote $[u]=M M$. If there are at least $\ell$ letters before, at least $r$ letters after the first occurrence of $x$ and $i<r$ letters after the second occurrence of $x$ in $u$, then we denote $[u]=M R_{j}$. If there are $i<r$ letters after the first occurrence of $x$ in $u$ and $j<r$ letters after the second occurrence of $x$, then we denote $[u]=R_{i} R_{j}$.

Observe that if $m \geq \ell+n+r-2$, then the number of patterns in the set $S_{m}$ does not depends on $m$ and is equal to $\ell(\ell-1) / 2+r(r-1) / 2+(\ell+1)(r+1)$. If $r=1$ then for each $m \geq l+n+r-2$ the set $S_{m}$ has only two patterns $M M$ and $M R_{0}$. If $\ell=0$ and $r>1$, then there are three types of word patterns in $S_{m}$, namely $M M, M R_{j}$ and $R_{i} R_{j}$. If $\ell=1$, then there are five types of patterns in $S_{m}$, namely $L_{i} M, L_{i} R_{j}$, $M M, M R_{j}$, and $R_{i} R_{j}$. If $\ell>1$, then there are six types of elements in $S_{m}$, namely $L_{i} L_{j}, L_{i} M, L_{i} R_{j}, M M, M R_{j}$, and $R_{i} R_{j}$.

For $k=\ell+n+r-2$ we consider a graph $G$ whose vertices are the word patterns that belong to $S_{k}$. If [ $\left.v_{1}\right],\left[v_{2}\right]$ are vertices of $G$, then we put an edge $\left\{\left[v_{1}\right],\left[v_{2}\right]\right\}$ if there is a pattern $[w] \in S_{k+1}$ such that $\left[v_{1}\right]<_{\mathfrak{B}_{\ell, r, n}}[w]$ and $\left[v_{2}\right]<_{\mathfrak{B}_{\ell, r, n}}[w]$. This property is definable in $F_{\infty} / \Leftrightarrow_{\mathfrak{B}_{\ell, r, n}}$. Moreover, we label the vertices [ $v$ ] of $G$ by the number of these $[w] \in S_{k+1}$ such that $[v]<_{\mathfrak{B}_{\ell, r, n}}[w]$. This property is also definable in $F_{\infty} / \Leftrightarrow_{\mathfrak{B}_{\ell, r, n}}$. It is easy to observe that the only vertex labeled 1 is $M M$. Hence, the word pattern $M M$ is definable in $S_{k}$ and, consequently, by Lemma 6.2 in $F_{\infty} / \Leftrightarrow_{\mathfrak{B}_{\ell, r, n}}$. The vertices labeled 2 have the forms $L_{i} L_{i+1}, L_{i} M, M R_{i}$, and $R_{i+1} R_{i}$. The rest are labeled 3 .

First consider the case when $\ell=0, n=r=2$. In this case $\left[x^{2} y\right]<_{\mathfrak{B}_{\ell, r, n}} M M$ and $\left[y x^{2}\right] \nless_{\mathfrak{B}_{\ell, r, n}} M M$, and therefore, both word patterns $\left[y x^{2}\right]$ and $\left[x^{2} y\right]$ are definable in $F_{\infty} / \Leftrightarrow_{\mathfrak{B}_{\ell, r, n}}$.

If $r=1$, then $G$ consists of two vertices $M M$ and $M R_{0}$. Hence, $M R_{0}$ is definable in $S_{k}$ and in $F_{\infty} / \Leftrightarrow_{\mathfrak{B}_{\ell, r, n}}$. In this case $\left[y x^{2}\right]<_{\mathfrak{B}_{\ell, r, n}} M R_{0}$ and $\left[x^{2} y\right] \nless_{\mathfrak{B}_{\ell, r, n}} M R_{0}$, and therefore, both word patterns $\left[y x^{2}\right]$ and $\left[x^{2} y\right]$ are definable in $F_{\infty} / \Leftrightarrow_{\mathfrak{B}, r, n}$.

Assume now that $r \geq 2$. Consider a subgraph $H$ of $G$ spanned by vertices that are labeled 1 and 2. The set of vertices of $H$ is obviously definable. It is clear that the distance in the graph $H$ between the vertex $M M$ and the vertices $L_{\ell-i} L_{\ell-i+1}$, $L_{\ell-i} M, M R_{r-i}$, and $R_{r-i+1} R_{r-i}$ is $i+1$. Since $r \geq 2$, there are exactly two vertices of $H$ with the distance to $M M$ equal to $r$. These vertices are $M R_{0}$ and $R_{1} R_{0}$. Hence, the set $\left\{M R_{0}, R_{1} R_{0}\right\}$ is definable in $S_{k}$. Moreover, for $R_{1} R_{0}$ there is only one word pattern $[v] \in S_{k-1}$ such that $[v]<_{\mathfrak{B}_{\ell, r, n}} R_{1} R_{0} \in S_{k}$. This word pattern is $R_{1} R_{0} \in$ $S_{k-1}$. But since $r \geq 2$ and $\ell+n>2$, for $M R_{0}$, there are at least two distinct patterns in $S_{k-1}$ with this property: the one that ends with $x y x$ and the one that ends with $x y z x$. Therefore, $R_{1} R_{0} \in S_{k}$ is definable in $F_{\infty} / \Leftrightarrow_{\mathfrak{B}_{\ell, r, n}}$. Now, $\left[y x^{2}\right]<_{\mathfrak{B}_{\ell, r, n}} R_{1} R_{0}$ and $\left[x^{2} y\right] \nless_{\mathfrak{B}_{\ell, r, n}} R_{1} R_{0}$. Thus, both word patterns [yx $\left.x^{2}\right]$ and $\left[x^{2} y\right]$ are definable in $F_{\infty} / \Leftrightarrow_{\mathfrak{B}_{\ell, r, n}}$. 


\section{Properties of varieties $\mathcal{V}_{a, b}^{-}$and $\mathcal{V}_{a, b}^{+}$}

Lemma 7.1 If $\mathfrak{A}$ is a set of 0 -reduced identities and $\mathfrak{R}$ is a set of regular identities, then $\operatorname{var}(\mathfrak{A} \cup \mathfrak{R}) \models u \approx v$ if and only if one of the following conditions holds:

(i) $u \sim \sim_{\mathfrak{R}} v$;

(ii) $u \geq_{\mathfrak{R}} a$ and $v \geq_{\mathfrak{R}} b$ for some possibly equal words $a$ and $b$ such that $\{a \approx 0$, $b \approx 0\} \subseteq \mathfrak{A}$.

Proof If one of the conditions (i) or (ii) holds, then clearly, $\operatorname{var}(\mathfrak{A} \cup \mathfrak{R}) \models u \approx v$.

Suppose now that $\operatorname{var}(\mathfrak{A} \cup \mathfrak{R}) \models u \approx v$. Consider a derivation of $u \approx v$ from $\mathfrak{A} \cup \mathfrak{R}$ :

$$
u \approx u_{1} \approx \cdots \approx u_{i} \approx u_{i+1} \approx \cdots v .
$$

If we cannot derive the identity $u \approx v$ from $\Re$, then we also need to use some identities from $\mathfrak{A}$. Let $u_{i}$ be the first place in this derivation where we use some identity $a \approx 0$ from $\mathfrak{A}$. Therefore, $u \approx u_{1} \approx \cdots \approx u_{i} \approx 0$. So, $u \geq_{\mathfrak{B}} a$. Similarly $v \geq_{\mathfrak{B}} b$ for some word $b$ such that $b \approx 0$ is in $\mathfrak{A}$.

Following [11], with each pair of possibly equal words $a$ and $b$ with $\operatorname{Cont}(a)=$ $\operatorname{Cont}(b)$ we associate two semigroup varieties:

$$
\mathcal{V}_{a, b}^{-}=\operatorname{var}\{v \approx u \approx 0 \mid v>a, u>b\}
$$

and

$$
\mathcal{V}_{a, b}^{+}=\operatorname{var}\{v \approx u \approx 0, p(a) \approx q(b) \mid v>a, u>b, p, q \in P(\operatorname{Cont}(a))\} .
$$

Here $P(\operatorname{Cont}(a))$ is the group of all permutations of $\operatorname{Cont}(a)$.

If $a=b$, then we set $\mathcal{V}_{a}^{-}:=\mathcal{V}_{a, b}^{-}$and $\mathcal{V}_{a}^{+}:=\mathcal{V}_{a, b}^{+}$.

Denote $\mathfrak{A}=\{v \approx 0 \mid v>a\} \cup\{u \approx 0 \mid u>b\}$ and $\mathfrak{C}=\{p(a) \approx q(b) \mid p, q \in$ $P(\operatorname{Cont}(a))\}$.

Lemma 7.2 Let $\mathfrak{B}$ be a set of balanced identities and let $a$ and $b$ be two words with $\operatorname{Cont}(a)=\operatorname{Cont}(b)$ that are either equal or incomparable modulo $\mathfrak{B}$ in the order $\leq_{\mathfrak{B}}$. Then

(i) the variety $\mathcal{V}_{a, b}^{+} \wedge \operatorname{var} \mathfrak{B}$ satisfies neither $a \approx 0$ nor $b \approx 0$;

(ii) if $\mathcal{V}_{a, b}^{+} \wedge \operatorname{var} \mathfrak{B} \models U \approx V$ and $U>_{\mathfrak{B}}$ a, then $\mathcal{V}_{a, b}^{+} \wedge \operatorname{var} \mathfrak{B} \models U \approx V \approx 0$ and $V>_{\mathfrak{B}}$ c for some $c \in\{a, b\}$.

Proof (i) Suppose that $\mathcal{V}_{a, b}^{+} \wedge \operatorname{var} \mathfrak{B} \models a \approx 0$. Let $a=V_{1} \approx V_{2} \approx \cdots \approx 0$ be a derivation of $a \approx 0$ from $\mathfrak{A} \cup \mathfrak{C} \cup \mathfrak{B}$. Since all identities in set $\mathfrak{C} \cup \mathfrak{B}$ are regular, we must use some identity from set $\mathfrak{A}$ in this derivation. Therefore, this derivation contains a word $V_{k}$ such that $V_{k}>a$ or $V_{k}>b$. Let $1<k<n$ be the least number with this property and suppose that $V_{k}>a$. So, we can consider another derivation of $a \approx 0$ : $a=V_{1} \approx V_{2} \approx \cdots \approx V_{k-1} \approx V_{k}=0$. This new derivation has a property that for each $i=1,2, \ldots, k-1$ the word $V_{i}$ is neither greater than $a$ nor greater than $b$. This 
means that either only identities from $\mathfrak{C}$ are used in this derivation or $a \sim_{\mathfrak{B}} V_{k}$. Let $j$ be the maximal number such that we apply an identity from $\mathfrak{C}$ to $V_{j}$ or $j=1$. Then $V_{j}=p(c)$ for some $c \in\{a, b\}$ and some renaming of variables $p$. Since $V_{j} \sim_{\mathfrak{B}} V_{k}$ we have that $p(c) \sim_{\mathfrak{B}} V_{k}$. Then by Lemma 5.3 we have that $V_{k} \Leftrightarrow_{\mathfrak{B}} c$. If $c=a$ then we obtain a contradiction with the fact that $V_{k}>a$. If $c=b \neq a$ then we obtain a contradiction with the fact that $a$ and $b$ are incomparable modulo $\mathfrak{B}$ in the order $\leq_{\mathfrak{B}}$. Thus, the variety $\mathcal{V}_{a, b}^{+} \wedge$ var $\mathfrak{B}$ does not satisfy $a \approx 0$.

Similarly, one can show that the variety $\mathcal{V}_{a, b}^{+} \wedge$ var $\mathfrak{B}$ does not satisfy $b \approx 0$.

(ii) If $U>_{\mathfrak{B}} a$, then by Lemma 5.3 one can find a word $W$ such that $U \sim_{\mathfrak{B}} W$ and $W>a$. Therefore, $\mathcal{V}_{a, b}^{+} \wedge \operatorname{var} \mathfrak{B} \models U \approx V \approx 0$. Since $\mathcal{V}_{a, b}^{+} \wedge$ var $\mathfrak{B} \models V \approx 0$, we can derive $V \approx 0$ from $\mathfrak{A} \cup \mathfrak{C} \cup \mathfrak{B}$. Let $V \approx V_{1} \approx V_{2} \approx \ldots \approx 0$ be a derivation of $V \approx 0$ from $\mathfrak{A} \cup \mathfrak{C} \cup \mathfrak{B}$. If $U_{i}$ is the first place in this derivation where we use some identity $\tau \notin \mathfrak{B}$, then two cases are possible:

Case 1: $\tau$ is $u \approx 0$ where $u>c$ for some $c \in\{a, b\}$. Then $U_{i} \geq u>c$ and by Lemma 5.3 we have that $V>_{\mathfrak{B}} c$.

Case 2: $\tau$ is $a \approx p(b)$ for some permutation $p$ of $\operatorname{Cont}(a)=\operatorname{Cont}(b)$. Then $U_{i}=$ $A \Theta(a) B$ for some word $A B$ and substitution $\Theta$. If the word $A B$ is empty and $\Theta$ is a renaming of variables, then $U_{i}=\Theta(a)$. But then $\mathcal{V}_{a, b}^{+} \wedge$ var $\mathfrak{B} \models \Theta(a) \approx 0$ which contradicts part (i). So, we can assume that the word $A B$ is not empty or $\Theta$ is not a renaming of variables, whence $U_{i}>a$. Then by Lemma 5.3 we have that $V>_{\mathfrak{B}} a$.

Case 3: $\tau$ is $b \approx q(b)$ for some permutation $q$ of $\operatorname{Cont}(b)$. This case is similar to Case 2 and the conclusion is that $V>_{\mathfrak{B}} b$.

Lemma 7.3 If $\mathfrak{B}$ is a set of balanced identities and $a$ and $b$ are two words with $\operatorname{Cont}(a)=\operatorname{Cont}(b)$ incomparable modulo $\mathfrak{B}$ in the order $\leq_{\mathfrak{B}}$, then

(i) $\mathcal{V}_{a}^{+}=\mathcal{V}_{a}^{-}$if and only if the word a is a power of some variable;

(ii) $\operatorname{var}\{a \approx 0, b \approx 0, \mathfrak{B}\} \subset \mathcal{V}_{a, b}^{+} \wedge \operatorname{var} \mathfrak{B} \subseteq \mathcal{V}_{a, b}^{-} \wedge \operatorname{var} \mathfrak{B} \subset \operatorname{var} \mathfrak{B}$.

Proof (i) If $a$ contains more than two variables, then the set $\{a \approx p(a) \mid p \in$ $P(\operatorname{Cont}(a))\}$ contains a non-trivial identity that cannot be derived from $\{v \approx 0 \mid v>a\}$.

(ii) The first inclusion is strict because the variety $\mathcal{V}_{a, b}^{+} \wedge$ var $\mathfrak{B}$ does not satisfy $a \approx 0$ by Lemma 7.2. The last inclusion is strict because the variety $\mathcal{V}_{a, b}^{-} \wedge \operatorname{var} \mathfrak{B}$ is periodic.

If $\mathfrak{B}$ is a set of identities, then an identity $\tau$ is called non-trivial modulo $\mathfrak{B}$ if $\tau$ does not follow from $\mathfrak{B}$. If $\mathfrak{B}$ contains only balanced identities we consider two types of identities $u \approx v$.

Type 1: $u \sim_{\mathfrak{B}} p(c)$ for some $c \in\{a, b\}$ and some permutation $p$ of $\operatorname{Cont}(a)$ and $v \sim_{\mathfrak{B}} q(c)$ for some $d \in\{a, b\}$ and some permutation $q$ of $\operatorname{Cont}(a)$.

Type 2: $u>_{\mathfrak{B}} c$ for some $c \in\{a, b\}$ and $v>_{\mathfrak{B}} d$ for some $d \in\{a, b\}$.

Lemma 7.4 Let $\mathfrak{B}$ be a set of balanced identities and let $a$ and $b$ be two words with $\operatorname{Cont}(a)=\operatorname{Cont}(b)$ that are either equal or incomparable modulo $\mathfrak{B}$ in the order $\leq \mathfrak{B}$. 
(i) $\mathcal{V} \supseteq \mathcal{V}_{a, b}^{-} \wedge \operatorname{var} \mathfrak{B}$ if and only if each non-trivial modulo $\mathfrak{B}$ identity of $\mathcal{V}$ has Type 2.

(ii) $\mathcal{V} \supseteq \mathcal{V}_{a, b}^{+} \wedge \operatorname{var} \mathfrak{B}$ if and only if each non-trivial modulo $\mathfrak{B}$ identity of $\mathcal{V}$ has Type 1 or Type 2.

Proof (i) Let $u \approx v$ be a non-trivial modulo $\mathfrak{B}$ identity of $\mathcal{V} \supseteq \mathcal{V}_{a, b}^{-} \wedge$ var $\mathfrak{B}$. Then by Lemma 7.1, $u \geq_{\mathfrak{B}} u^{\prime}$ and $v \geq_{\mathfrak{B}} v^{\prime}$ where $u^{\prime}>c$ for some $c \in\{a, b\}$ and $v^{\prime}>d$ for some $d \in\{a, b\}$. Thus, by Lemma 5.3 we have that $u>_{\mathfrak{B}} c$ and $v>_{\mathfrak{B}} d$. Therefore, the identity $u \approx v$ is of Type 2 .

(ii) Let $u \approx v$ be a non-trivial modulo $\mathfrak{B}$ identity of $\mathcal{V} \supseteq \mathcal{V}_{a, b}^{+} \wedge \operatorname{var} \mathfrak{B}$.

If $u$ or $v$ is incomparable neither with $a$ nor with $b$ modulo $\mathfrak{B}$, then $u \approx v$ is trivial modulo $\mathfrak{B}$. If $u>_{\mathfrak{B}} c$ for some $c \in\{a, b\}$, then by Lemma 7.2 we have that $v>_{\mathfrak{B}} d$ for some $d \in\{a, b\}$. In this case the identity $u \approx v$ is of Type 2 . If the identity $u \approx v$ is irregular, then say, $v$ contains some variable $x$ but $u$ does not. If we replace $x$ by $a$, then we obtain an identity $u \approx w$ such that $w>a$. Then in view of Lemma 7.2, we have that $u>_{\mathfrak{B}} a$. This takes us to the previous case. If the identity $u \approx v$ is regular and $u \Leftrightarrow_{\mathfrak{B}} v$, then the identity $u \approx v$ is of Type 1 by Lemma 5.3.

Lemma 7.5 Let $\mathfrak{B}$ be a set of balanced identities and let $a$ and $b$ be two words with $\operatorname{Cont}(a)=\operatorname{Cont}(b)$ that are either equal or incomparable modulo $\mathfrak{B}$ in the order $\leq_{\mathfrak{B}}$. If $\tau$ is a non-trivial identity modulo $\mathfrak{B}$, then $\mathcal{V}_{a, b}^{+} \wedge \operatorname{var} \mathfrak{B} \models \tau$ if and only if $\tau$ has Type 1 or Type 2.

Proof If $\mathcal{V}_{a, b}^{+} \wedge \operatorname{var} \mathfrak{B} \models \tau$, then by Lemma 7.4 the identity $\tau$ has either Type 1 or Type 2. If $\tau$ is of Type 1 , then $\tau$ follows from $\{p(a) \approx q(b) \mid p, q \in P(\operatorname{Cont}(a))\}$. If $u \approx v$ is of Type 2, then by Lemma 5.3 we have that $u \sim_{\mathfrak{B}} u^{\prime}$ and $u^{\prime}>c$. So, $\mathcal{V}_{a, b}^{+} \wedge \operatorname{var} \mathfrak{B} \models u \approx 0$. Similarly, $\mathcal{V}_{a, b}^{+} \wedge \operatorname{var} \mathfrak{B} \models v \approx 0$. Therefore, $\mathcal{V}_{a, b}^{+} \wedge \operatorname{var} \mathfrak{B} \models$ $u \approx v$.

Lemma 7.6 Let $\mathfrak{B}$ be a set of balanced identities and let $a$ and $b$ be two words with $\operatorname{Cont}(a)=\operatorname{Cont}(b)$ that are either equal or incomparable modulo $\mathfrak{B}$ in the order $\leq_{\mathfrak{B}}$. Then the variety $\mathcal{V}_{a, b}^{+} \wedge \operatorname{var} \mathfrak{B}$ is a cover of the variety $\operatorname{var}\{a \approx 0, b \approx 0, \mathfrak{B}\}$. In particular, the variety $\mathcal{V}_{a}^{+}$is a cover of the variety $\operatorname{var}\{a \approx 0\}$.

Proof Let $\mathcal{V}$ be a variety such that $\operatorname{var}\{a \approx 0, b \approx 0, \mathfrak{B}\} \subseteq \mathcal{V} \subset \mathcal{V}_{a, b}^{+} \wedge$ var $\mathfrak{B}$. Since $\mathcal{V}$ is properly contained in $\mathcal{V}_{a}^{+} \wedge \operatorname{var} \mathfrak{B}$, the variety $\mathcal{V}$ satisfies some identity $u \approx v$ such that $\mathcal{V}_{a}^{+} \wedge \operatorname{var} \mathfrak{B}$ does not satisfy $u \approx v$. Since the variety $\mathcal{V}$ contains $\operatorname{var}\{a \approx 0$, $b \approx 0, \mathfrak{B}\}$, Lemma 7.1 implies that $u \geq_{\mathfrak{B}} c$ for some $c \in\{a, b\}$ and $v \geq_{\mathfrak{B}} d$ for some $d \in\{a, b\}$. In view of Lemma 7.5, two essentially different cases are possible.

Case 1: $u>_{\mathfrak{B}} c$ for some $c \in\{a, b\}$ and $v \sim_{\mathfrak{B}} p(a)$ for some renaming of variables $p$. Since $\mathcal{V}_{a, b}^{+} \wedge \operatorname{var} \mathfrak{B}$ satisfies $u \approx 0$, we have that $\mathcal{V} \models p(a) \approx 0$. Since $\mathcal{V}_{a, b}^{+} \wedge \operatorname{var} \mathfrak{B}$ satisfies $b \approx p(a)$, we have that $\mathcal{V} \models b \approx 0$. Therefore $\mathcal{V}=\operatorname{var}\{a \approx 0$, $b \approx 0, \mathfrak{B}\}$.

Case 2: $u \sim_{\mathfrak{B}} p(a)$ for some renaming of variables $p$ and $v \sim_{\mathfrak{B}} q(c)$ for some $c \in\{a, b\}$ and some renaming of variables $q$ such that the identity $u \approx v$ is irregular. Without loss of generality, $\mathcal{V}$ satisfies $p(a) \approx v$ such that $v$ contains some letter $x$ that 
does not occur in $p(a)$. If we replace $x$ by $a$, we obtain an identity $p(a) \approx v^{\prime}$ such that $v^{\prime}>a$. Since $\mathcal{V}_{a}^{+} \wedge \operatorname{var} \mathfrak{B}$ satisfies $v^{\prime} \approx 0$, we have that $\mathcal{V} \models p(a) \approx 0$. Since $\mathcal{V}_{a, b}^{+} \wedge \operatorname{var} \mathfrak{B}$ satisfies $b \approx p(a)$, we have that $\mathcal{V} \models b \approx 0$. Therefore $\mathcal{V}=\operatorname{var}\{a \approx 0$, $b \approx 0, \mathfrak{B}\}$.

The next technical lemma is needed only to justify Corollary 7.1.

Lemma 7.7 Let $\mathfrak{B}$ be a set of balanced identities and let $a$ and $b$ be two words with $\operatorname{Cont}(a)=\operatorname{Cont}(b)$ that are incomparable modulo $\mathfrak{B}$ in the order $\leq_{\mathfrak{B}}$. Let $\mathcal{V}$ be $a$ variety that contains $\operatorname{var}\{a \approx 0, b \approx 0, \mathfrak{B}\}$ and is contained in $\mathcal{V}_{a, b}^{-} \wedge \operatorname{var} \mathfrak{B}$. If $\mathcal{V}$ does not contain $\mathcal{V}_{a, b}^{+} \wedge \operatorname{var} \mathfrak{B}$, then $\mathcal{V} \models a \approx 0$ or $\mathcal{V} \models b \approx 0$.

Proof Since $\mathcal{V}$ does not contain $\mathcal{V}_{a, b}^{+} \wedge$ var $\mathfrak{B}$, the variety $\mathcal{V}$ satisfies some identity $u \approx v$ such that $\mathcal{V}_{a}^{+} \wedge \operatorname{var} \mathfrak{B}$ does not satisfy $u \approx v$. Since the variety $\mathcal{V}$ contains $\operatorname{var}\{a \approx 0, b \approx 0, \mathfrak{B}\}$, Lemma 7.1 implies that $u \geq_{\mathfrak{B}} c$ for some $c \in\{a, b\}$ and $v \geq_{\mathfrak{B}} d$ for some $d \in\{a, b\}$. In view of Lemma 7.5, two essentially different cases are possible.

Case 1: $u>_{\mathfrak{B}} c$ for some $c \in\{a, b\}$ and $v \sim_{\mathfrak{B}} p(a)$ for some renaming of variables $p$. By Lemma 5.3 one can find a word $w$ such that $u \sim_{\mathfrak{B}} w$ and $w>c$. Therefore, $\mathcal{V} \models w \approx p(a)$. Since $w>c$ and $\mathcal{V} \subseteq \mathcal{V}_{a, b}^{-} \wedge \operatorname{var} \mathfrak{B}$ we have that $\mathcal{V} \models w \approx 0$. So, the variety $\mathcal{V}$ satisfies the identity $p(a) \approx 0$ and consequently $a \approx 0$.

Case 2: $u \sim_{\mathfrak{B}} p(a)$ for some renaming of variables $p$ and $v \sim_{\mathfrak{B}} q(c)$ for some $c \in\{a, b\}$ and some renaming of variables $q$ such that the identity $u \approx v$ is irregular. Without loss of generality, $\mathcal{V}$ satisfies $p(a) \approx v$ such that $v$ contains some letter $x$ that does not occur in $p(a)$. If we replace $x$ by $a$, we obtain an identity $p(a) \approx v^{\prime}$ such that $v^{\prime}>a$. Since $v^{\prime}>a$ and $\mathcal{V} \subseteq \mathcal{V}_{a, b}^{-} \wedge \operatorname{var} \mathfrak{B}$, we have that $\mathcal{V} \models v^{\prime} \approx 0$. So, the variety $\mathcal{V}$ satisfies the identity $p(a) \approx 0$ and consequently $a \approx 0$.

If we consider cases symmetric to Case 1 and Case 2 , then we obtain that $\mathcal{V} \models b \approx 0$.

Corollary 7.1 Let $\mathfrak{B}$ be a set of balanced identities and let $a$ and $b$ be two words with $\operatorname{Cont}(a)=\operatorname{Cont}(b)$ incomparable modulo $\mathfrak{B}$ in the order $\leq_{\mathfrak{B}}$. Then the variety $\mathcal{V}_{a, b}^{+} \wedge \operatorname{var} \mathfrak{B}$ is the only cover of the variety $\operatorname{var}\{a \approx 0, b \approx 0, \mathfrak{B}\}$ that is contained in $\mathcal{V}_{a, b}^{-} \wedge \operatorname{var} \mathfrak{B}$ but in neither $\operatorname{var}\{a \approx 0, \mathfrak{B}\}$ nor $\operatorname{var}\{b \approx 0, \mathfrak{B}\}$.

The next lemma follows from Lemma 1.3(iii) in [27].

Lemma 7.8 [27] If a permutative nil-variety satisfies an identity of the form $u \approx v$ with $u<v$, then $\mathcal{V}$ also satisfies the identity $u \approx 0$.

The next technical lemma is needed only to justify Corollary 7.2.

Lemma 7.9 Let $\mathfrak{B}$ be a set of balanced identities that contains a permutation identity. Let $\mathcal{V}$ be a nil-variety that contains $\operatorname{var}\{a \approx 0, \mathfrak{B}\}$ and is contained in var $\mathfrak{B}$. If $\mathcal{V}$ does not contain $\mathcal{V}_{a}^{+} \wedge \operatorname{var} \mathfrak{B}$, then $\mathcal{V} \models a \approx 0$. 
Proof Since $\mathcal{V}$ does not contain $\mathcal{V}_{a}^{+} \wedge$ var $\mathfrak{B}$, the variety $\mathcal{V}$ satisfies some identity $u \approx v$ such that $\mathcal{V}_{a}^{+} \wedge \operatorname{var} \mathfrak{B}$ does not satisfy $u \approx v$. Since $\mathcal{V}$ contains the variety $\operatorname{var}\{a \approx 0, \mathfrak{B}\}$, Lemma 7.1 implies that $u \geq_{\mathfrak{B}} a$ and $v \geq_{\mathfrak{B}} a$. In view of Lemma 7.5, two essentially different cases are possible.

Case 1: $u>_{\mathfrak{B}} a$ and $v \sim_{\mathfrak{B}} p(a)$ for some renaming of variables $p$. By Lemma 5.3 one can find a word $w$ such that $u \sim_{\mathfrak{B}} w$ and $w>a$. Therefore, $\mathcal{V} \models w \approx p(a)$. Since $w>a$ we have that $w>p(a)$. Since $\mathcal{V}$ is a permutative nil-variety, by Lemma 7.8, the variety $\mathcal{V}$ satisfies the identity $p(a) \approx 0$ and consequently $a \approx 0$.

Case 2: $u \sim_{\mathfrak{B}} p(a)$ and $v \sim_{\mathfrak{B}} q(a)$ for some renamings of variables $p$ and $q$ such that the identity $u \approx v$ is irregular. Without loss of generality, $\mathcal{V}$ satisfies $p(a) \approx v$ such that $v$ contains some letter $x$ that does not occur in $p(a)$. If we replace $x$ by $p(a)$, we obtain an identity $p(a) \approx v^{\prime}$ such that $v^{\prime}>p(a)$. Since $\mathcal{V}$ is a permutative nilvariety, by Lemma 7.8 , the variety $\mathcal{V}$ satisfies the identity $p(a) \approx 0$ and consequently $a \approx 0$.

Corollary 7.2 Let $\mathfrak{B}$ be a set of balanced identities that contains a permutation identity and let $a$ be a word. Then the variety $\mathcal{V}_{a}^{+} \wedge$ var $\mathfrak{B}$ is the only cover of the variety $\operatorname{var}\{a \approx 0, \mathfrak{B}\}$ in the lattice $L(\operatorname{var} \mathfrak{B}) \cap \mathbf{N}$.

\section{The set of varieties of the form $\operatorname{var}\{u \approx 0, \mathfrak{B}\}$ is definable in $L(\operatorname{var} \mathfrak{B})$}

If $\mathfrak{A}$ is a set of 0 -reduced identities and $\mathfrak{B}$ is a set of regular identities, then we say that $\mathfrak{A}$ is reduced modulo $\mathfrak{B}$ if every two words $a$ and $b$ with $\{a \approx 0, b \approx 0\} \subseteq \mathfrak{A}$ are incomparable in the order $\leq_{\mathfrak{B}}$.

Proposition 8.1 For a nil-variety $\mathcal{V}$ the following conditions are equivalent.

(i) $\mathcal{V}$ is b0-reduced.

(ii) $\mathcal{V}=\operatorname{var}(\mathfrak{A} \cup \mathfrak{B})$ for some set of balanced identities $\mathfrak{B}$ and some non-empty set of 0 -reduced identities $\mathfrak{A}$.

(iii) $\mathcal{V}=\operatorname{var}(\mathfrak{A} \cup \mathfrak{B})$ for some set of balanced identities $\mathfrak{B}$ and some non-empty set of 0 -reduced identities $\mathfrak{A}$ reduced modulo $\mathfrak{B}$.

(iv) $\mathcal{V}$ can be defined by an irredundant basis of balanced and 0 -reduced identities.

Proof (i) $\rightarrow$ (ii). Since $\mathcal{V}$ is $b 0$-reduced, each unbalanced identity $u \approx v$ of $\mathcal{V}$ can be derived from $u \approx 0$ and $v \approx 0$. If $\mathfrak{A}$ is the set of all 0 -reduced identities satisfied by $\mathcal{V}$ and $\mathfrak{B}$ is the set of all balanced identities satisfied by $\mathcal{V}$, then $\mathcal{V}=\operatorname{var}(\mathfrak{A} \cup \mathfrak{B})$.

(ii) $\rightarrow$ (iii). Let us show that one can find $\mathfrak{A}^{\prime} \subseteq \mathfrak{A}$ such that $\mathfrak{A}^{\prime}$ is reduced modulo $\mathfrak{B}$ and $\operatorname{var}(\mathfrak{A} \cup \mathfrak{B})=\operatorname{var}\left(\mathfrak{A}^{\prime} \cup \mathfrak{B}\right)$. The following argument is a modification of the argument of Martynova [14]. For each $n>0$, let $\mathfrak{A}_{n}$ denote the set of all identities $u \approx 0$ in $\mathfrak{A}$ such that $|u| \leq n$. Since for each $n>0$ the set $\mathfrak{A}_{n}$ is finite, we can reduce $\mathfrak{A}_{n}$ modulo $\mathfrak{B}$ to $\mathfrak{A}_{n}^{\prime}$ by throwing away extra identities of length $n$. Since set $\mathfrak{B}$ contains only balanced identities, no derivation of any 0-reduced identity $a \approx 0$ uses any 0-reduced identity $b \approx 0$ with $|b|>|a|$. So, the set $\mathfrak{A}^{\prime}=\bigcup_{n>0} \mathfrak{A}_{n}^{\prime}$ is reduced modulo $\mathfrak{B}$. Evidently, $\operatorname{var}(\mathfrak{A} \cup \mathfrak{B})=\operatorname{var}\left(\mathfrak{A}^{\prime} \cup \mathfrak{B}\right)$. 
(iii) $\rightarrow$ (iv) follows from Proposition 4.1 in [20] that says that each set of balanced and 0 -reduced identities is equivalent to its irreducible subset.

Evidently (iv) $\rightarrow$ (iii) $\rightarrow$ (ii).

(ii) $\rightarrow$ (i). Let $u \approx v$ be an unbalanced identity of $\mathcal{V}$. Consider a derivation of $u \approx v$ from $\mathfrak{A} \cup \mathfrak{B}:$

$$
u \approx u_{1} \approx \cdots \approx u_{i} \approx u_{i+1} \approx \cdots v .
$$

In view of Lemma 3.3(iii) we cannot derive $u \approx v$ only from $\mathfrak{B}$. Let $u_{i}$ be the first place in this derivation where we use some identity in $\mathfrak{A}$. Then $\mathcal{V} \models u \approx u_{i} \approx 0$. Therefore, the variety $\mathcal{V}$ is $b 0$-reduced.

Recall that a variety that can be defined by 0 -reduced identities only is called a 0 -reduced variety. Obviously, each 0 -reduced variety is also a $b 0$-reduced variety. By a result of Ježek [4], there are 0-reduced varieties with infinite irredundant identity bases. In contrast, Proposition 8.1 and a result by Perkins [15] imply the following.

Lemma 8.1 Each permutative b0-reduced variety can be defined by a finite basis of balanced and 0-reduced identities.

Lemma 8.2 Let $\mathfrak{B}$ be a set of balanced identities and let $\mathfrak{A}$ be a set of 0 -reduced identities that is reduced modulo $\mathfrak{B}$ and contains at least two identities. Then

(i) $\operatorname{var}(\mathfrak{A} \cup \mathfrak{B})=\mathcal{V}_{1} \wedge \mathcal{V}_{2}$ for some incomparable varieties $\mathcal{V}_{1} \in L(\operatorname{var} \mathfrak{B}) \cap \mathbf{N}$ and $\mathcal{V}_{2} \in L(\operatorname{var} \mathfrak{B}) \cap \mathbf{N}$

(ii) if $\mathfrak{B}$ contains a permutation identity, then the variety $\operatorname{var}(\mathfrak{A} \cup \mathfrak{B})$ has at least two covers in $L(\operatorname{var} \mathfrak{B}) \cap \mathbf{N}$.

Proof (i) Let $b \approx 0$ and $c \approx 0$ be two distinct identities in $\mathfrak{A}$. It is easy to see that $\mathcal{V}=\operatorname{var}((\mathfrak{A} \backslash\{b \approx 0\}) \cup \mathfrak{B}) \wedge \operatorname{var}((\mathfrak{A} \backslash\{c \approx 0\}) \cup \mathfrak{B})$. Since the set $\mathfrak{A}$ is reduced modulo $\mathfrak{B}$, the varieties $\mathcal{V}_{1}=\operatorname{var}((\mathfrak{A} \backslash\{b \approx 0\}) \cup \mathfrak{B})$ and $\mathcal{V}_{2}=\operatorname{var}((\mathfrak{A} \backslash\{c \approx 0\}) \cup \mathfrak{B})$ are incomparable.

(ii) Since $\mathfrak{B}$ contains a permutation identity, each variety in $L(\operatorname{var} \mathfrak{B})$ is permutative. Since each variety in $L(\operatorname{var} \mathfrak{B}) \cap \mathbf{N}$ is permutative and periodic, by the result of Perkins [15], every variety in $L(\operatorname{var} \mathfrak{B}) \cap \mathbf{N}$ is finitely based. Therefore, there are no infinite descending chains between $\mathcal{V}_{1}$ and $\mathcal{V}=\operatorname{var}(\mathfrak{A} \cup \mathfrak{B})$ or between $\mathcal{V}_{2}$ and $\mathcal{V}$. Thus, $\mathcal{V}$ has both a cover contained in $\mathcal{V}_{1}$ and a cover contained in $\mathcal{V}_{2}$; these two covers cannot coincide since $\mathcal{V}_{1} \wedge \mathcal{V}_{2}=\mathcal{V}$. We conclude that $\mathcal{V}$ must have at least two covers in $L(\operatorname{var} \mathfrak{B}) \cap \mathbf{N}$.

Lemma 8.3 Let a be a word and let $\mathfrak{B} \cup \mathfrak{C}$ be a set of balanced identities such that the set $\mathfrak{C}$ contains an identity that does not follow from $\mathfrak{B} \cup\{a \approx 0\}$. Then

(i) $\operatorname{var}\{a \approx 0, \mathfrak{B} \cup \mathfrak{C}\}=\mathcal{V}_{1} \wedge \mathcal{V}_{2}$ for some incomparable varieties $\mathcal{V}_{1} \in L(\operatorname{var} \mathfrak{B}) \cap \mathbf{N}$ and $\mathcal{V}_{2} \in L(\operatorname{var} \mathfrak{B}) \cap \mathbf{N}$;

(ii) if $\mathfrak{B}$ contains a permutation identity, then the variety $\operatorname{var}\{a \approx 0, \mathfrak{B} \cup \mathfrak{C}\}$ has at least two covers in $L(\operatorname{var} \mathfrak{B}) \cap \mathbf{N}$. 
Proof (i) The variety $\mathcal{V}_{a}^{+} \wedge \operatorname{var}(\mathfrak{B} \cup \mathfrak{C})$ is a cover of $\operatorname{var}\{a \approx 0, \mathfrak{B} \cup \mathfrak{C}\}$ by Lemma 7.6. Therefore, $\operatorname{var}\{a \approx 0, \mathfrak{B} \cup \mathfrak{C}\}=\operatorname{var}\{a \approx 0, \mathfrak{B}\} \wedge \mathcal{V}_{a}^{+} \wedge \operatorname{var}(\mathfrak{B} \cup \mathfrak{C})$. The variety $\mathcal{V}_{a}^{+} \wedge$ $\operatorname{var}(\mathfrak{B} \cup \mathfrak{C})$ is not contained in $\operatorname{var}\{a \approx 0, \mathfrak{B}\}$ because it does not satisfy $a \approx 0$.

(ii) Using a similar argument as in Lemma 8.2(ii), one can show that there is a cover of $\operatorname{var}\{a \approx 0, \mathfrak{B} \cup \mathfrak{C}\}$ under $\operatorname{var}\{a \approx 0, \mathfrak{B}\}$ as well as a cover under $\mathcal{V}_{a}^{+} \wedge$ $\operatorname{var}(\mathfrak{B} \cup \mathfrak{C})$, and these two covers cannot coincide.

Proposition 8.2 Let $\mathfrak{B}$ be a set of balanced identities that contains a permutation identity. A b0-reduced variety $\mathcal{V} \in L(\operatorname{var} \mathfrak{B})$ has exactly one cover in $L$ (var $\mathfrak{B}) \cap \mathbf{N}$ if and only if $\mathcal{V}=\operatorname{var}\{u \approx 0, \mathfrak{B}\}$.

Proof If $\mathcal{V}=\operatorname{var}\{u \approx 0, \mathfrak{B}\}$, then $\mathcal{V}$ has exactly one cover in $L(\operatorname{var} \mathfrak{B}) \cap \mathbf{N}$ by Corollary 7.2.

Now assume that $\mathcal{V}$ has exactly one cover in $L(\operatorname{var} \mathfrak{B}) \cap \mathbf{N}$. Since $\mathcal{V}$ is a $b 0$-reduced variety in $L(\operatorname{var} \mathfrak{B})$, we have that $\mathcal{V}=\operatorname{var}(\mathfrak{A} \cup \mathfrak{B} \cup \mathfrak{C})$ for some set of 0 -reduced identities $\mathfrak{A}$ and some set of balanced identities $\mathfrak{C}$. In view of Proposition 8.1, we can assume that the set $\mathfrak{A}$ is reduced modulo $\mathfrak{B} \cup \mathfrak{C}$. If $\mathfrak{A}$ contains two identities then, by Lemma 8.2, $\mathcal{V}$ must have at least two covers in $L$ (var $\mathfrak{B} \cup \mathfrak{C}) \cap \mathbf{N}$ and consequently, in $L(\operatorname{var} \mathfrak{B}) \cap \mathbf{N}$.

To avoid a contradiction, we must assume that $\mathfrak{A}$ contains exactly one identity, i.e. $\mathcal{V}=\operatorname{var}\{u \approx 0, \mathfrak{B} \cup \mathfrak{C}\}$ for some word $u$. If the set $\mathfrak{C}$ contains some identity that does not follow from $\mathfrak{B} \cup\{a \approx 0\}$, then by Lemma 8.3, the variety $\mathcal{V}$ must have at least two covers in $L(\operatorname{var} \mathfrak{B}) \cap \mathbf{N}$. We conclude that $\mathcal{V}=\operatorname{var}\{u \approx 0, \mathfrak{B}\}$.

Lemma 2.4, Theorem 4.1 and Proposition 8.2 imply the following.

Theorem 8.1 Let $\mathfrak{B}$ be a set of balanced identities that contains a permutation identity. Then the set of all varieties of the form $\operatorname{var}\{u \approx 0, \mathfrak{B}\}$ is definable in L(var $\mathfrak{B})$.

\section{Some definable and semi-definable varieties and sets of varieties in $L$ (var $\mathfrak{B})$}

If $\mathfrak{B}$ is a set of balanced identities, then a $b 0$-reduced variety $\mathcal{V} \in L(\operatorname{var} \mathfrak{B})$ is called $\mathfrak{B}-0$-reduced if every balanced identity of $\mathcal{V}$ follows from $\mathfrak{B}$. Since every balanced identity follows from $x y \approx y x$, the set of all $\{x y \approx y x\}$-0-reduced varieties coincides with the set of all commutative $b 0$-reduced varieties. The set of all $\{x \approx x\}$-0-reduced varieties obviously coincides with the set of all 0-reduced varieties.

It is proved in [11] and reproved in [24, 29] that the set of all 0-reduced varieties is definable in SEM. Corollary 2.12 in [24] (reproduced as [25, Theorem 2.3]) and Proposition 2.2 in [18] define the set of all 0-reduced varieties and the set of all commutative $b 0$-reduced varieties as follows.

Lemma 9.1 If $\mathfrak{B}=\{x \approx x\}$ or $\mathfrak{B}=\{x y \approx y x\}$, then a variety $\mathcal{V} \in L(\operatorname{var} \mathfrak{B})$ is $\mathfrak{B}-0$ reduced if and only if $\mathcal{V}$ is a nil-variety and $\mathcal{V}$ is lower-modular in $L(\operatorname{var} \mathfrak{B})$.

Lemma 9.1 defines the set of all commutative $b 0$-reduced varieties in a different way than Corollary 4.1. 
Question 9.1 Is it true that the set of all $\mathfrak{B}$-0-reduced varieties is definable in $L(\operatorname{var} \mathfrak{B})$ for every set of balanced identities $\mathfrak{B}$ ?

The following definition is essentially the definition of semi-definability in [11] extended to arbitrary self-dual sets of regular identities.

Definition 9.1 Let $\mathfrak{B}$ be a self-dual set of regular identities and $\mathcal{V} \in L$ (var $\mathfrak{B})$. We say that $\mathcal{V}$ is definable in $L(\operatorname{var} \mathfrak{B})$ up to $\operatorname{var}\left\{x^{2} y \approx 0, \mathfrak{B}\right\}$ if there exists a first-order formula $\Phi_{\mathcal{V}}(\bar{x}, \bar{y})$ that turns into a true statement on $L(\operatorname{var} \mathfrak{B})$ if and only if $\bar{x}=\mathcal{V}$ and $\bar{y}=\operatorname{var}\left\{x^{2} y \approx 0, \mathfrak{B}\right\}$, or else $\bar{x}=\mathcal{V}^{\delta}$ and $\bar{y}=\operatorname{var}\left\{y x^{2} \approx 0, \mathfrak{B}\right\}$.

Lemma 9.2 Let $\mathfrak{B}$ be a self-dual set of regular identities and $\mathcal{V}, \mathcal{U} \in L(\operatorname{var} \mathfrak{B})$. If each of the varieties $\mathcal{V}$ and $\mathcal{U}$ is definable in $L(\operatorname{var} \mathfrak{B})$ up to $\operatorname{var}\left\{x^{2} y \approx 0, \mathfrak{B}\right\}$, then the variety $\mathcal{V} \wedge \mathcal{U}$ is also definable in $L(\operatorname{var} \mathfrak{B})$ up to $\operatorname{var}\left\{x^{2} y \approx 0, \mathfrak{B}\right\}$.

Proof Let $\Phi_{u}(\bar{x}, \bar{y})$ and $\Phi_{v}(\bar{x}, \bar{y})$ be the formulas that define the varieties $\mathcal{V}$ and $\mathcal{U}$ in $L(\operatorname{var} \mathfrak{B})$ up to $\operatorname{var}\left\{x^{2} y \approx 0, \mathfrak{B}\right\}$. Then the formula

$$
(\forall \bar{z}, \bar{p})\left(\Phi_{u}(\bar{z}, \bar{y}) \& \Phi_{v}(\bar{p}, \bar{y}) \longrightarrow(\bar{x}=\bar{z} \wedge \bar{p})\right)
$$

turns into a true statement on $L(\operatorname{var} \mathfrak{B})$ if and only if $\bar{x}=\mathcal{V} \wedge \mathcal{U}$ and $\bar{y}=\operatorname{var}\left\{x^{2} y \approx\right.$ $0, \mathfrak{B}\}$, or else $\bar{x}=\mathcal{V}^{\delta} \wedge \mathcal{U}^{\delta}$ and $\bar{y}=\operatorname{var}\left\{y x^{2} \approx 0, \mathfrak{B}\right\}$.

For the rest of this article we focus on definability in $L(\operatorname{var} \mathfrak{B})$, where $\mathfrak{B}=\mathfrak{B}_{\ell, r, n}$ for some $\ell, r \geq 0$ and $n \geq 2$. But each result on definability in this section and in the next section holds true for an arbitrary set of balanced identities $\mathfrak{B}$ that satisfies the following conditions:

(i) $\mathfrak{B}$ contains a permutation identity.

(ii) Each word pattern modulo $\mathfrak{B}$ is definable (or semi-definable) in $F_{\infty} / \Leftrightarrow_{\mathfrak{B}}$.

In particular, in view of Corollary 6.1 , each statement on definability up to $\operatorname{var}\left\{x^{2} y \approx 0, \mathfrak{B}\right\}$ in Sects. 9 and 10 holds true if $\mathfrak{B}$ is an arbitrary self-dual set of balanced identities with $\ell(\mathfrak{B})=r(\mathfrak{B})>0$ that contains a permutation identity.

Theorem 9.1 Let $\mathfrak{B}=\mathfrak{B}_{\ell, r, n}$ for some $\ell, r \geq 0$ and $n>1$.

(i) If $\ell=r$, then each $\mathfrak{B}-0$-reduced variety is definable in $L$ (var $\mathfrak{B})$ up to $\operatorname{var}\left\{x^{2} y \approx\right.$ $0, \mathfrak{B}\}$.

(ii) If $\ell \neq r$, then each $\mathfrak{B}-0$-reduced variety is definable in $L$ (var $\mathfrak{B})$.

Proof (i) First, we assume that $\mathcal{V}=\operatorname{var}\{u \approx 0, \mathfrak{B}\}$ for some word $u$. By Corollaries 6.1 and 8.1 the variety $\mathcal{V}$ is definable in $L(\operatorname{var} \mathfrak{B})$ up to $\operatorname{var}\left\{x^{2} y \approx 0, \mathfrak{B}\right\}$. Now if $\mathcal{V}$ is an arbitrary $\mathfrak{B}-0$-reduced variety, then by Lemma 8.1 , we have that $\mathcal{V}=\operatorname{var}(\mathfrak{A} \cup \mathfrak{B})$ for some finite set of 0 -reduced identities $\mathfrak{A}$. The rest follows from Lemma 9.2.

(ii) follows from Corollaries 6.2, 8.1 and Lemma 8.1 in a similar manner.

Theorem 9.1 implies the following partial answer to Question 9.1. 
Corollary 9.1 Let $\mathfrak{B}=\mathfrak{B}_{\ell, r, n}$ for some $\ell, r \geq 0$ and $n>1$ and let $m>0$, then the set of all $\mathfrak{B}-0$-reduced varieties contained in $\operatorname{var}\left\{x^{m} \approx 0, \mathfrak{B}\right\}$ is definable in L(var $\left.\mathfrak{B}\right)$.

Proof If $\mathcal{V} \subseteq \operatorname{var}\left\{x^{m} \approx 0, \mathfrak{B}\right\}$, then $\mathcal{V}=\operatorname{var}(\mathfrak{A} \cup \mathfrak{B})$ for some set of 0-reduced identities $\mathfrak{A}$. In view of Proposition 8.1 we may assume that the set $\mathfrak{A}$ is reduced modulo $\mathfrak{B}$. By the result of Perkins [15], the number of identities in $\mathfrak{A}$ is bounded by a computable function of $\ell, r, n$ and $m$.

Corollary 9.2 Let $\mathfrak{B}=\mathfrak{B}_{\ell, r, n}$ for some $\ell, r \geq 0$ and $n>1$ and let $a$ and $b$ be two words with $\operatorname{Cont}(a)=\operatorname{Cont}(b)$ that are either equal or incomparable in the order $\leq_{\mathfrak{B}}$.

(i) If $\ell=r$, then each of the varieties $\mathcal{V}_{a, b}^{-} \wedge \operatorname{var} \mathfrak{B}$ and $\mathcal{V}_{a, b}^{+} \wedge \operatorname{var} \mathfrak{B}$ is definable in $L(\operatorname{var} \mathfrak{B})$ up to $\operatorname{var}\left\{x^{2} y \approx 0, \mathfrak{B}\right\}$.

(ii) If $\ell \neq r$, then each of the varieties $\mathcal{V}_{a, b}^{-} \wedge \operatorname{var} \mathfrak{B}$ and $\mathcal{V}_{a, b}^{+} \wedge \operatorname{var} \mathfrak{B}$ is definable in $L(\operatorname{var} \mathfrak{B})$.

Proof (i) The variety $\mathcal{V}_{a, b}^{-} \wedge \operatorname{var} \mathfrak{B}$ is definable in $L(\operatorname{var} \mathfrak{B})$ up to $\operatorname{var}\left\{x^{2} y \approx 0, \mathfrak{B}\right\}$ as a $\mathfrak{B}$-0-reduced variety by Theorem 9.1 . The variety $\mathcal{V}_{a}^{+} \wedge$ var $\mathfrak{B}$ is definable in $L(\operatorname{var} \mathfrak{B})$ up to $\operatorname{var}\left\{x^{2} y \approx 0, \mathfrak{B}\right\}$ as the only cover of a $\mathfrak{B}$-0-reduced variety in $L(\operatorname{var} \mathfrak{B}) \cap \mathbf{N}$ by Corollary 7.2 and Lemma 2.4. The variety $\mathcal{V}_{a, b}^{+} \wedge$ var $\mathfrak{B}$ is definable in $L(\operatorname{var} \mathfrak{B})$ up to $\operatorname{var}\left\{x^{2} y \approx 0, \mathfrak{B}\right\}$ by the formula in Corollary 7.1.

(ii) Similar.

If $\mathfrak{B}$ is a set of balanced identities and $a$ and $b$ are two possibly equal words with $\operatorname{Cont}(a)=\operatorname{Cont}(b)$, then $\operatorname{Sub}(\mathfrak{B}, a, b)$ denotes the class of all varieties of the form $\operatorname{var}(\mathfrak{B} \cup\{p(c) \approx q(d) \mid c, d \in\{a, b\}, p, q \in G(\operatorname{Cont}(a))\})$ where $G(\operatorname{Cont}(a))$ is a non-trivial group of permutations of $\operatorname{Cont}(a)$. If $a=b$ then we $\operatorname{set} \operatorname{Sub}(\mathfrak{B}, a):=$ $\operatorname{Sub}(\mathfrak{B}, a, b)$.

The following theorem is a translation of Theorems 5.1 and 5.4 in [12].

Theorem 9.2 Let $\mathfrak{B}=\mathfrak{B}_{\ell, r, n}$ for some $\ell, r \geq 0$ and $n>1$ and let $a$ and $b$ are two words with $\operatorname{Cont}(a)=\operatorname{Cont}(b)$ that are either equivalent or incomparable in the order $\leq_{\mathfrak{B}}$. If $\ell=r$ [respectively $\left.\ell \neq r\right]$, then the class of varieties $\operatorname{Sub}(\mathfrak{B}, a, b)$ is definable up to $\operatorname{var}\left\{x^{2} y \approx 0, \mathfrak{B}\right\}$ [respectively definable] in $L$ (var $\left.\mathfrak{B}\right)$ by the following formula:

$\mathcal{V} \in \operatorname{Sub}(\mathfrak{B}, a, b)$ if and only if $\mathcal{V} \supseteq \mathcal{V}_{a, b}^{+} \wedge \operatorname{var} \mathfrak{B}$, and if $\mathcal{V}=\mathcal{V}_{1} \wedge \mathcal{V}_{2}$ and $\mathcal{V}_{2} \supseteq$ $\mathcal{V}_{a, b}^{-} \wedge \operatorname{var} \mathfrak{B}$, then $\mathcal{V}_{1}=\mathcal{V}$.

Proof If $\mathcal{V} \in \operatorname{Sub}(\mathfrak{B}, a, b)$, then $\mathcal{V} \supseteq \mathcal{V}_{a, b}^{+} \wedge \operatorname{var} \mathfrak{B}$ by Lemma 7.4. Now assume that $\mathcal{V}=\mathcal{V}_{1} \wedge \mathcal{V}_{2}$ where $\mathcal{V}_{2} \supseteq \mathcal{V}_{a, b}^{-} \wedge$ var $\mathfrak{B}$. Since $\mathcal{V}_{2} \supseteq \mathcal{V}_{a}^{-} \wedge$ var $\mathfrak{B}$, by Lemma 7.4 the variety $\mathcal{V}_{2}$ satisfies only identities of Type 2 . Since $\mathcal{V}=\mathcal{V}_{1} \wedge \mathcal{V}_{2}$ and no identity of Type 1 can be derived from the identities of Type 2 and $\mathfrak{B}$, the variety $\mathcal{V}_{1}$ must satisfy all identities of Type 1 that are satisfied by $\mathcal{V}$, i.e. $\mathcal{V}_{1} \subseteq \mathcal{V}$. Therefore, $\mathcal{V}_{1}=\mathcal{V}$.

Now we assume that a variety $\mathcal{V}$ satisfies this formula. Since $\mathcal{V} \supseteq \mathcal{V}_{a, b}^{+} \wedge$ var $\mathfrak{B}$, by Lemma 7.4 the variety $\mathcal{V}$ satisfies only identities of Types 1 and 2 . So, we have that 
$\mathcal{V}=\operatorname{var}\left(\mathfrak{B} \cup T_{1} \cup T_{2}\right)$ where $T_{1}$ is a finite set of identities of Type 1 and $T_{2}$ is a finite set of identities of Type 2 .

Since the variety var $\mathfrak{B}$ does not satisfy the rest of the formula, we conclude that the set $T_{1} \cup T_{2}$ is not empty. Since for non-empty $T_{2}$ the variety $\operatorname{var}\left(\mathfrak{B} \cup T_{2}\right)$ does not satisfy the rest of the formula, we conclude that the set $T_{1}$ is not empty.

Now the goal is to show that set $T_{2}$ is empty. If set $T_{2}$ is not-empty, then $\mathcal{V}=$ $\operatorname{var}\left(\mathfrak{B} \cup T_{1}\right) \wedge \operatorname{var}\left(\mathfrak{B} \cup T_{2}\right)$ and the variety $\mathcal{V}$ does not satisfy the rest of the formula for $\mathcal{V}_{1}=\operatorname{var}\left(\mathfrak{B} \cup T_{1}\right)$ and $\mathcal{V}_{2}=\operatorname{var}\left(\mathfrak{B} \cup T_{2}\right)$. To avoid the contradiction, we must assume that the set $T_{2}$ is empty. Therefore, $\mathcal{V}=\operatorname{var}\left(\mathfrak{B} \cup T_{1}\right) \in \operatorname{Sub}(\mathfrak{B}, a, b)$.

The rest follows from Corollary 9.2.

\section{Definability of each variety of the form $\operatorname{var}\{u \approx v, \mathfrak{B}\}$ in $L(\operatorname{var} \mathfrak{B})$ if $u \approx v$ is a regular identity and the words $u$ and $v$ are either equivalent or incomparable in the order $\leq_{\mathfrak{B}}$}

If $\mathfrak{B}$ is a set of balanced identities then $|\mathfrak{B}|$ denotes the length of the shortest nontrivial identity from $\mathfrak{B}$. In view of Lemma 5.2, any two words that are shorter than $|\mathfrak{B}|$ and are equivalent (incomparable) in the order $\leq_{\mathfrak{B}}$ are also equivalent (incomparable) in the order $\leq$.

Lemma 10.1 Let $\mathfrak{B}=\mathfrak{B}_{\ell, r, n}$ for some $\ell, r \geq 0$ and $n>1$ and let $u$ be a word with $|\operatorname{Cont}(u)|=k>1$ and $|u|<|\mathfrak{B}|$. If $\ell=r$ [respectively $\ell \neq r]$, then the set $\operatorname{Sub}_{p p}(\mathfrak{B}, u)$ of all varieties of the form $\operatorname{var}\{u \approx p(u), \mathfrak{B}\}$, where the order of the permutation $p$ is a prime power, is definable up to $\operatorname{var}\left\{x^{2} y \approx 0, \mathfrak{B}\right\}$ [respectively definable] in $L(\operatorname{var} \mathfrak{B})$.

Proof Since $|u|<|\mathfrak{B}|$, the lattice $\operatorname{Sub}(\mathfrak{B}, u)$ is anti-isomorphic to the lattice of all subgroups of the symmetric group $\mathbb{S}_{k}$. We use the fact that, for every $k>1$, each subgroup of $\mathbb{S}_{k}$, which is not cyclic of prime power order, has at least two incomparable subgroups. Therefore the set $\operatorname{Sub}_{p p}(\mathfrak{B}, u)$ is defined in $\operatorname{Sub}(\mathfrak{B}, u)$ as the set of all varieties $\mathcal{V} \in \operatorname{Sub}(\mathfrak{B}, u)$ such that there are no varieties $\mathcal{V}_{1}$ and $\mathcal{V}_{2}$ in $\operatorname{Sub}(\mathfrak{B}, u)$ satisfying $\mathcal{V} \leq \mathcal{V}_{1} \wedge \mathcal{V}_{2}$ and neither $\mathcal{V}_{1} \subseteq \mathcal{V}_{2}$ nor $\mathcal{V}_{2} \subseteq \mathcal{V}_{1}$. The rest follows from Theorem 9.2.

Recall (see Sect. 5) that there are two types of elementary substitutions that rename variables and we agreed to use the term renaming of variables to refer to a one-toone renaming of variables. If $x$ and $y$ are two distinct variables then $E_{x=y}$ denotes the elementary substitution that renames $y$ by $x$ and is identical on all other variables.

Lemma 10.2 Let $\mathfrak{B}$ be a set of balanced identities and let $u$ be $a$ word such that $|u|<|\mathfrak{B}|$. Let $p$ be a permutation of $\operatorname{Cont}(u)=\{s, t, \ldots\}$. If $\mathcal{V}=\operatorname{var}\{u \approx p(u), \mathfrak{B}\}$, then

(i) $\left[E_{s=t}(u)\right] \mathcal{V}=\left\{E_{s=t}\left(p^{\ell}(u)\right) \mid \ell>0\right\}$;

(ii) any two words in the equivalence class $\left[E_{s=t}(u)\right] \mathcal{V}$ have the same content and are either equivalent or incomparable in the order $\leq_{\mathfrak{B}}$. 
Proof (i) Obviously, the equivalence class $\left[E_{s=t}(u)\right] \mathcal{V}$ contains each word of the form $E_{s=t}\left(p^{\ell}(u)\right)$. Since $|u|=|p(u)|<|\mathfrak{B}|$, each identity of the form $E_{s=t}(u) \approx W$ follows only from $u \approx p(u)$. In order to show that each word $W$ in the equivalence class $\left[E_{s=t}(u)\right] \mathcal{V}$ is of the required form, it is enough to check that if we derive an identity of the form $E_{s=t}\left(p^{\ell}(u)\right) \approx W$ from $u \approx p(u)$ in one step, then $W=E_{s=t}\left(p^{m}(u)\right)$ for some $m>0$. If $E_{s=t}\left(p^{\ell}(u)\right) \approx W$ follows from $u \approx p(u)$ in one step, then without any loss we can assume that $E_{s=t}\left(p^{\ell}(u)\right)=\Theta(u)$ and $W=\Theta(p(u))$. Since $\left|E_{s=t}\left(p^{\ell}(u)\right)\right|=|u|$, the substitution $\Theta$ is a composition of some length-preserving elementary substitutions. Without any loss, we have that $\Theta=E_{s=t} q$ for some renaming of variables $q$ invariant on $\operatorname{Cont}(u)$. Therefore, we have that $E_{s=t}\left(p^{\ell}(u)\right)=E_{s=t} q(u)$ and, consequently, $p^{\ell}=q$. So, $W=p^{\ell+1}(u)$ is of the required form.

(ii) Clearly, for any $\ell>0$ and $m>0$ the words $E_{s=t}\left(p^{\ell}(u)\right)$ and $E_{s=t}\left(p^{m}(u)\right)$ have the same content. Suppose that $E_{s=t}\left(p^{\ell}(u)\right) \leq_{\mathfrak{B}} E_{s=t}\left(p^{m}(u)\right)$. Since $\left|E_{s=t}\left(p^{l}(u)\right)\right|=\left|E_{s=t}\left(p^{m}(u)\right)\right|<|\mathfrak{B}|$, this can only happen if $E_{s=t}\left(p^{m}(u)\right)=$ $\Theta\left(E_{s=t}\left(p^{\ell}(u)\right)\right)$ where $\Theta$ is a composition of renamings and equalizings of variables. But since $\operatorname{Cont}\left(E_{s=t}\left(p^{\ell}(u)\right)\right)=\operatorname{Cont}\left(E_{s=t}(p(u))\right)$, the substitution $\Theta$ can only be a renaming of variables. Thus, the words $E_{s=t}\left(p^{\ell}(u)\right)$ and $E_{s=t}\left(p^{m}(u)\right)$ are either equivalent or incomparable in the order $\leq_{\mathfrak{B}}$.

Lemma 10.3 Let $u$ be $a$ word and let $p$ and $q$ be two permutations of $\operatorname{Cont}(u)=$ $\{x, y, s, t, \ldots\}$ such that $x \neq y$ and $s \neq t$. Then the following are equivalent:

(i) $E_{s=t}(q(u))=p\left(E_{x=y}(u)\right)$;

(ii) $q=p, p(y)=t$ and $p(x)=s$.

Proof (i) $\rightarrow$ (ii). Both words $E_{s=t}(q(u))$ and $p\left(E_{x=y}(u)\right)$ are obtained from $u$ by renaming variables in $u$. Since the word $E_{s=t}(p(u))$ does not contain $t$ and the word $p\left(E_{x=y}(u)\right)$ does not contain $p(y)$, we must have that $p(y)=t$. The variables $x$ and $y$ are the only variables in $u$ that receive that same name $p(x)$ in $p\left(E_{x=y}(u)\right)$. Since $E_{s=t}(q(u))=p\left(E_{x=y}(u)\right)$, the same variables are identified (as $\left.s\right)$ in $E_{s=t}(q(u))$. Therefore $p(x)=s$.

If a variable $z$ is different from $y$, then $q(z)$ stands in the same positions in $E_{s=t}(q(u))$ as $z$ does in $u$. Since the variable $p(z)$ stands in the same positions in $p\left(E_{x=y}(u)\right)$ as $z$ does in $u$, we have that $q(z)=p(z)$. Since both renamings $p$ and $q$ are one-to one and invariant on $\operatorname{Cont}(u)$, we also have that $q(y)=p(y)=t$. Since the renamings $p$ and $q$ coincide on all variables in $\operatorname{Cont}(u)$, we have that $q=p$.

(ii) $\rightarrow$ (i) can be easily verified.

Let $\mathfrak{B}$ be a set of balanced identities, $u$ a word, and $p$ a permutation of $\operatorname{Cont}(u)$ of prime power order. Then for each distinct $x, y \in \operatorname{Cont}(u)$ we define set $V(\mathfrak{B}, u, p, x, y)$ as the set of all varieties $\operatorname{var}\{u \approx q(u), \mathfrak{B}\} \in \operatorname{Sub}_{p p}(\mathfrak{B}, u)$ such that $q^{\ell}(x)=p(x)$ and $q^{\ell}(y)=p(y)$ for some $\ell>0$. Obviously, $\operatorname{var}\{u \approx p(u), \mathfrak{B}\} \in$ $V(\mathfrak{B}, u, p, x, y) \subseteq \operatorname{Sub}_{p p}(\mathfrak{B}, u)$.

Lemma 10.4 Let $\mathfrak{B}=\mathfrak{B}_{\ell, r, n}$ for some $\ell, r \geq 0$ and $n>1$, let $u$ be a word such that $|u|<|\mathfrak{B}|$ and let $p$ be a permutation of $\operatorname{Cont}(u)$ of a prime power order. If $\ell=r$ 
$[$ respectively $\ell \neq r]$, then for each distinct $x, y \in \operatorname{Cont}(u)$ the set $V(\mathfrak{B}, u, p, x, y)$ is definable up to $\operatorname{var}\left\{x^{2} y \approx 0, \mathfrak{B}\right\}$ [respectively definable] in $L(\operatorname{var} \mathfrak{B})$ as the set of all varieties $\mathcal{V} \in \operatorname{Sub}_{p p}(\mathfrak{B}, u)$ such that $\mathcal{V}$ is contained in some variety that belongs to the set $\operatorname{Sub}\left(\mathfrak{B}, E_{s=t}(u), E_{s=t}(p(u))\right)$ where $s=p(x)$ and $t=p(y)$.

Proof Suppose that $\mathcal{V}=\operatorname{var}\{u=q(u), \mathfrak{B}\} \in \operatorname{Sub}_{p p}(\mathfrak{B}, u)$ such that $q^{\ell}(x)=p(x)$ and $q^{\ell}(y)=p(y)$ for some $\ell>0$. Since $\mathcal{V} \models v \approx q^{\ell}(v)$, the variety $\mathcal{V}$ also satisfies $E_{s=t}(u) \approx E_{s=t}\left(q^{\ell}(u)\right)$ for $s=p(x)$ and $t=p(y)$.

Therefore, in view of Lemma 10.3 , the variety $\mathcal{V}$ satisfies

$$
\begin{aligned}
E_{s=t}(u) & \approx E_{s=t}\left(q^{\ell}(u)\right)=q^{\ell}\left(E_{x=y}(u)\right) \\
& =q^{\ell} p^{-1} p\left(E_{x=y}(u)\right)=q^{\ell} p^{-1}\left(E_{s=t}(p(u))\right) .
\end{aligned}
$$

So, $\mathcal{V}$ is contained in a variety that lies in the $\operatorname{set} \operatorname{Sub}\left(\mathfrak{B}, E_{s=t}(v), E_{s=t}(p(u))\right)$.

If $\mathcal{V}=\operatorname{var}\{u \approx q(u), \mathfrak{B}\} \in \operatorname{Sub}_{p p}(\mathfrak{B}, u)$ is contained in some variety that belongs to the $\operatorname{set} \operatorname{Sub}\left(\mathfrak{B}, E_{s=t}(v), E_{s=t}(p(u))\right)$ where $s=p(x)$ and $t=p(y)$, then $\mathcal{V}$ satisfies the identity $E_{s=t}(u) \approx \tau\left(E_{s=t}(p(u))\right)$ for some renaming of variables $\tau$ invariant on $\operatorname{Cont}(u) \backslash t$. Then by Lemmas 10.2 and 10.3 we have that $\tau\left(E_{s=t}(p(u))\right)=$ $\tau p\left(E_{x=y}(u)\right)=E_{s=t}\left(q^{\ell}(u)\right)$ for some $\ell>0$. Now by Lemma 10.3 we have that $q^{\ell}=\tau p, q^{\ell}(x)=s$ and $q^{\ell}(y)=t$. Therefore, $\mathcal{V}$ belongs to the set $V(\mathfrak{B}, u, p, x, y)$.

The rest follows from Theorem 9.2 and Lemmas 10.1 and 10.2.

If $\mathfrak{B}$ is a set of balanced identities, $u$ is a word and $p$ is a permutation, then we denote $\mathcal{V}_{p}=\operatorname{var}\{u \approx p(u), \mathfrak{B}\}$. If $\mathcal{V}_{p} \in \operatorname{Sub}_{p p}(\mathfrak{B}, u)$, then $\operatorname{Sub}_{p p}\left(\mathcal{V}_{p}\right)$ denotes the set of all varieties $\mathcal{V}_{q} \in \operatorname{Sub}_{p p}(\mathfrak{B}, u)$ that satisfy the following condition:

(*) for each $x \neq y$ and $s \neq t$, there exists an $\ell>0$ such that $p^{\ell}(x, y)=(s, t)$ if and only if one can find an $m>0$ such that $q^{m}(x, y)=(s, t)$.

Obviously, for each $x \neq y$ we have that $\mathcal{V}_{p} \in \operatorname{Sub}_{p p}\left(\mathcal{V}_{p}\right) \subseteq V(\mathfrak{B}, u, p, x, y) \subseteq$ $\operatorname{Sub}_{p p}(\mathfrak{B}, u)$.

Lemma 10.5 Let $\mathfrak{B}=\mathfrak{B}_{\ell, r, n}$ for some $\ell, r \geq 0$ and $n>1$, let $u$ be $a$ word and let $p$ be a permutation of $\operatorname{Cont}(u)$ of a prime power order. If $\ell=r$ [respectively $\ell \neq r$ ], then the set $\operatorname{Sub}_{p p}\left(\mathcal{V}_{p}\right)$ is definable up to $\operatorname{var}\left\{x^{2} y \approx 0, \mathfrak{B}\right\}$ [respectively definable] in $L(\operatorname{var} \mathfrak{B})$ as the set of all varieties $\mathcal{V} \in \operatorname{Sub}_{p p}(\mathfrak{B}, u)$ that satisfy the following condition:

$(* *)$ for each $x \neq y \in \operatorname{Cont}(u)$ and each permutation $q$, we have that $\mathcal{V} \in$ $V(\mathfrak{B}, u, q, x, y)$ if and only if $\mathcal{V}_{p} \in V(\mathfrak{B}, u, q, x, y)$.

Proof Suppose that $\mathcal{V}_{\tau} \in \operatorname{Sub}_{p p}\left(\mathcal{V}_{p}\right)$. Take $x \neq y \in \operatorname{Cont}(u)$ and some permutation $q$ such that $q(x)=s$ and $q(y)=t$.

If $\mathcal{V}_{\tau} \in V(\mathfrak{B}, u, q, x, y)$, then for some $\ell>0$ we have that $\tau^{\ell}(x, y)=(s, t)$. Since $\mathcal{V}_{\tau} \in \operatorname{Sub}_{p p}\left(\mathcal{V}_{p}\right)$, one can find an $m$ such that $p^{m}(x, y)=(s, t)$. Therefore, $\mathcal{V}_{p} \in$ $V(\mathfrak{B}, u, q, x, y)$. 
Conversely, if $\mathcal{V}_{p} \in V(\mathfrak{B}, u, q, x, y)$, then for some $\ell>0$ we have that $p^{\ell}(x, y)=$ $(s, t)$. Since $\mathcal{V}_{\tau} \in \operatorname{Sub}_{p p}\left(\mathcal{V}_{p}\right)$, one can find an $m$ such that $\tau^{m}(x, y)=(s, t)$. Therefore, $\mathcal{V}_{\tau} \in V(\mathfrak{B}, u, q, x, y)$.

Now suppose that variety $\mathcal{V}_{\tau} \in \operatorname{Sub}_{p p}(\mathfrak{B}, u)$ satisfies Condition (**). In order to prove that $\mathcal{V}_{\tau} \in \operatorname{Sub}_{p p}\left(\mathcal{V}_{p}\right)$ we take $x \neq y$ and $s \neq t$.

If one can find an $\ell>0$ such that $p^{\ell}(x, y)=(s, t)$, then $\mathcal{V}_{p^{\ell}} \in V\left(\mathfrak{B}, u, p^{\ell}, x, y\right)$ and by Condition $(* *)$ we have that $\mathcal{V}_{\tau} \in V\left(\mathfrak{B}, u, p^{\ell}, x, y\right)$. Therefore, one can find an $m>0$ such that $\tau^{m}(x, y)=(s, t)$.

Conversely, if one can find an $m>0$ such that $\tau^{m}(x, y)=(s, t)$, then $\mathcal{V}_{\tau^{m}} \in$ $V\left(\mathfrak{B}, u, \tau^{m}, x, y\right)$ and by Condition $(* *)$ we have that $\mathcal{V}_{p} \in V\left(\mathfrak{B}, u, \tau^{m}, x, y\right)$. Therefore, one can find an $\ell>0$ such that $p^{\ell}(x, y)=(s, t)$.

The rest follows from Lemma 10.4.

Theorem 10.1 Let $\mathfrak{B}=\mathfrak{B}_{\ell, r, n}$ for some $\ell, r \geq 0$ and $n>1$, let $u$ be a word such that $|u|<|\mathfrak{B}|$, and let $p$ be a permutation of $\operatorname{Cont}(u)$. If $\ell=r$ [respectively $\ell \neq r]$, then the variety $\operatorname{var}\{u \approx p(u), \mathfrak{B}\}$ is definable up to $\operatorname{var}\left\{x^{2} y \approx 0, \mathfrak{B}\right\}$ [respectively definable] in $L(\mathfrak{B})$.

Proof We follow the direction suggested in [3, Sect. 10]. First, we prove Theorem 10.1 for the case when the permutation $p$ has a prime power order. In this case the variety $\mathcal{V}_{p}=\operatorname{var}\{u \approx p(u), \mathfrak{B}\}$ is contained in the set $\operatorname{Sub}_{p p}\left(\mathcal{V}_{p}\right)$ which is definable up to $\operatorname{var}\left\{x^{2} y \approx 0, \mathfrak{B}\right\}$ [respectively definable] in $L(\mathfrak{B})$ by Lemma 10.5 . Our goal is to show that the variety $\mathcal{V}_{p}$ is the only variety in this set.

Assume that $|\operatorname{Cont}(u)|=k>1$. Let $\operatorname{Sub}_{p p}$ denote the set of all cyclic subgroups of $\mathbb{S}_{k}$ of prime power orders. Then the variety $\mathcal{V}_{p}$ corresponds to the cyclic subgroup $\langle p\rangle \in \operatorname{Sub}_{p p}$. Instead of the set $\operatorname{Sub}_{p p}\left(\mathcal{V}_{p}\right)$ we consider the corresponding set $P(p)$ that consists of all permutations $q \in \mathrm{Sub}_{p p}$ that satisfy the following condition:

$(\dagger)$ for each $i \neq j$ and $s \neq t$, there exists an $\ell>0$ such that $p^{\ell}(i, j)=(s, t)$ if and only if one can find an $m>0$ such that $q^{m}(i, j)=(s, t)$.

The task of proving that the variety $\mathcal{V}_{p}$ is the only variety in the $\operatorname{set} \operatorname{Sub}_{p p}\left(\mathcal{V}_{p}\right)$ is equivalent to the task of proving that $P(p)=\langle p\rangle$. In each of the following lemmas we narrow the set of possible members of $P(p)$.

Let $Z_{1}, \ldots, Z_{\ell}$ be the orbits of the fixed permutation $p$.

Lemma 10.6 If $\langle q\rangle \subset P(p)$, then the orbits of $q$ are exactly $Z_{1}, \ldots, Z_{\ell}$.

Proof If the orbits of $q$ are not the same as the orbits of $p$, then there are $i \neq s$ that belong to the same orbit of $p$ but do not belong to the same orbit of $q$ (or visa versa). This means that $p^{r}(i)=s$ for some $r$ but there is no $m>0$ such that $q^{m}(i)=s$. Therefore, Condition $(\dagger)$ is violated.

The next restriction concerns the cyclic structure of the permutation $q$. Let $c_{1} \cdots c_{\ell}$ be the cyclic decomposition of our fixed permutation $p$, where $c_{i}$ is a cyclic permutation of $Z_{i}$. 
Lemma 10.7 If $\langle q\rangle \subset P(p)$, then $q$ acts as the product $c_{1}^{t_{1}} \cdots c_{l}^{t_{\ell}}$ of powers of the cycles $c_{1}, \ldots, c_{\ell}$ such that $c_{i}$ and $c_{i}^{t_{i}}$ generate the same cyclic group for each $i$. (It is equivalent to say that $q$ leaves every $Z_{i}$ invariant and the group generated by $q$, restricted to $Z_{i}$, is $\left\langle c_{i}\right\rangle$ ).

Proof By Lemma 10.6, since $\langle q\rangle \subset P(p)$, the permutation $q$ acts on each $Z_{i}$ as some cycle (the trivial cycle if $\left|Z_{i}\right|=1$ ). If an orbit $Z_{i}$ has less than four elements then there is a unique transitive cyclic group on $Z_{i}$, hence every $\langle q\rangle \subset P(p)$ has the required property with respect to such an orbit $Z_{i}$. Now consider an orbit $Z_{i}$ with more than three elements.

Choose $s \in Z_{i}$. Then $q$ and $c_{i}$ generate the same cyclic group on $Z_{i}$ if and only if for every $j$ there is an $\ell>0$ such that $q^{\ell}(s)=c_{i}^{j}(s)$ and $q^{\ell}\left(c_{i}(s)\right)=c_{i}^{j+1}(s)$.

Since this is true for $q=p$, by the definition of $P(p)$, this is also true for every $q$ such that $\langle q\rangle \subset P(p)$.

Now, if all $Z_{i}$ are one-element sets, then $p$ is the identity permutation. Hence, in the sequel, we can assume that one of $Z_{i}$ contains at least two elements. Let $\gamma$ be the prime appearing in the order of $p$.

Our next restrictions concern the exponents $t_{i}$ in the cyclic decomposition $q=$ $c_{1}^{t_{1}} \cdots c_{\ell}^{t_{\ell}}$ where $\langle q\rangle \subset P(p)$. We may assume that, for all $i, \gamma$ does not divide $t_{i}$, $1 \leq t_{i}<\left|c_{i}\right|$ when $\left|c_{i}\right|>1$, and $t_{i}=1$ if $\left|c_{i}\right|=1$.

Lemma 10.8 Let $\langle q\rangle \subset P(p)$ and suppose that $q=c_{1}^{t_{1}} \cdots c_{\ell}^{t_{\ell}}$.If $\left|c_{i}\right|=\left|c_{j}\right|$ for some $i, j$, then $t_{i}=t_{j}$.

Proof Assume to the contrary that $t_{i} \neq t_{j}$. Let us denote $N=\left|c_{i}\right|=\gamma^{m}$. By renumbering, we can assume that $c_{i}=(1, \ldots, N)$ and $c_{j}=(N+1, \ldots 2 N)$. Obviously, there exists some $\ell>0$ such that $p^{\ell}(1)=2$ and $p^{\ell}(N+1)=N+2$ Since $\langle q\rangle \subset P(p)$, there exists $d$ such that $q^{d}(1)=2$, and $q^{d}(N+1)=N+2$. Therefore, $c_{i}^{d t_{i}}(1)=2$ and $c_{j}^{d t_{j}}(N+1)=N+2$. Since $c_{i}$ acts regularly on the set $Z_{i}$, we have $d t_{i} \equiv 1(\bmod N)$. But then $d t_{j} \not \equiv 1(\bmod N)$, as $1 \leq t_{i}, t_{j}<N, t_{i} \neq t_{j}$ and $t_{i}$ and $t_{j}$ are prime to $N$. Finally, since $c_{j}$ acts regularly on the set $Z_{j}$, we have $c_{j}^{d t_{j}}(N+1) \neq N+2$, which leads to a contradiction and thus proves Lemma 10.8.

We may without loss of generality assume that for every $i<\ell,\left|c_{i}\right| \leq\left|c_{i+1}\right|$. Let $\left\langle p_{1}\right\rangle \subset P(p)$. Note that we can choose $d$ such that $p_{1}^{d}$ acts as $c_{1}$ on $Z_{1}$. If $p_{2}=p_{1}^{d}$ then since $d$ is prime to $\gamma$, we have that $\left\langle p_{2}\right\rangle=\left\langle p_{1}\right\rangle \subset P(p)$. We choose $p_{2}$ from $\left\{\sigma:\langle\sigma\rangle=\left\langle p_{2}\right\rangle\right\}$ such that in the representation $p_{2}=c_{1}^{t_{1}} \cdots c_{\ell}^{t_{\ell}}, t_{1}=t_{2}=\cdots=t_{j}=1$ for $j$ as large as possible. As the final step we prove

Lemma 10.9 If $\left\langle p_{2}\right\rangle \subset P(p)$, then $p_{2}=p$.

Proof Assume to the contrary $p_{2} \neq p$. Let $i$ be minimal with $t_{i} \neq 1$. We know from the observation above that $i>1$. Assume that the permutations $c_{i-1}$ and $c_{i}$ act on the sets $\{1, \ldots, N\}$ and $\{s+1, \ldots, s+M\}$, respectively, where $N=\left|c_{i-1}\right|=\gamma^{m_{i-1}}$, and $M=\left|c_{i}\right|=\gamma^{m_{i}}$. We follow the proof of the previous lemma: $p(1)=2, p(s+1)=$ 
$s+2$. Since $\left\langle p_{2}\right\rangle \in P(p)$, there is $d$ such that $p_{2}^{d}(1)=2$ and $p_{2}^{d}(s+1)=s+2$. This means that $c_{i-1}^{t_{i-1} d}(1)=2$ and $c_{i}^{t_{i} d}(s+1)=s+2$. Since $t_{i-1}=1$, we have, according to the first equality, that $d \equiv 1\left(\bmod \left|c_{i-1}\right|\right)$, where $\left|c_{i-1}\right|=\gamma^{m_{i-1}}$. Since $\left|c_{j}\right|$ divides $\left|c_{j+1}\right|$ for all $j$, we have $d \equiv 1\left(\bmod \left|c_{j}\right|\right) ; j<i$. In particular $\gamma$ does not divide $d$, whence $\left\langle p_{2}^{d}\right\rangle=\left\langle p_{2}\right\rangle$. Moreover, the second equality implies that $t_{i} d \equiv 1$ $\left(\bmod \left|c_{i}\right|\right)$, and consequently $t_{i} d \equiv 1\left(\bmod \left|c_{j}\right|\right)$ for all $j \leq i$. Thus we can replace $p_{2}$ by $p_{3}=p_{2}^{d}$ such that

$$
p_{3}=c_{1} \cdots c_{i} c_{i+1}^{s_{i+1}} \cdots c_{\ell}^{s_{\ell}},
$$

for some $s_{i+1}, \ldots, s_{\ell}$. This contradicts our maximality assumption for $p_{2}$.

Now, it follows from Lemma 10.9 that $P(p)=\langle p\rangle$ and Theorem 10.1 is proved for the case when the order of $p$ is a prime power. Otherwise, the order of permutation $p$ is $n_{1} n_{2} \cdots n_{k}$ where for each $1 \leq i \leq k$ the number $n_{i}$ is a prime power. In this case the variety $\mathcal{V}_{p}=\operatorname{var}\{u \approx p(u), \mathfrak{B}\}$ is definable up to $\operatorname{var}\left\{x^{2} y \approx 0, \mathfrak{B}\right\}$ [respectively definable] in $L(\operatorname{var} \mathfrak{B})$ by the formula $\mathcal{V}_{p}=\mathcal{V}_{n_{1}} \cap \cdots \cap \mathcal{V}_{n_{k}}$ and Lemma 9.2. This completes the proof of Theorem 10.1.

Theorem 10.2 Let $\mathfrak{B}=\mathfrak{B}_{\ell, r, n}$ for some $\ell, r \geq 0$ and $n>1$ and let $u$ and $v$ be words with $\operatorname{Cont}(u)=\operatorname{Cont}(v)$, of lengths shorter than $|\mathfrak{B}|$, and incomparable in the order $\leq_{\mathfrak{B}}$. If $\ell=r$ [respectively $\left.\ell \neq r\right]$, then the variety $\operatorname{var}\{u \approx v, \mathfrak{B}\}$ is definable up to $\operatorname{var}\left\{x^{2} y \approx 0, \mathfrak{B}\right\}$ [respectively definable] in $L(\operatorname{var} \mathfrak{B})$.

Proof We start with the following.

Lemma 10.10 Let $\mathfrak{B}=\mathfrak{B}_{\ell, r, n}$ for some $\ell, r \geq 0$ and $n>1$ and let $u$ and $v$ be words with $\operatorname{Cont}(u)=\operatorname{Cont}(v)$, of lengths shorter than $|\mathfrak{B}|$, and incomparable in the order

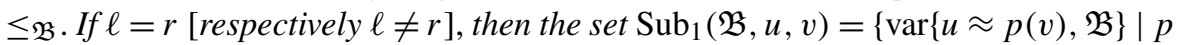
is a permutation of $\operatorname{Cont}(u)\}$ is definable up to $\operatorname{var}\left\{x^{2} y \approx 0, \mathfrak{B}\right\}$ [respectively definable] in $L(\operatorname{var} \mathfrak{B})$.

Proof Denote $\mathcal{V}=\{u \approx p(v), \mathfrak{B}\}$. Since the words $u$ and $v$ are shorter than $|\mathfrak{B}|$ and incomparable in the order $\leq_{\mathfrak{B}}$, the equivalence class $[u]_{\mathcal{V}}$ contains only $u$ and $p(v)$. Therefore, the set $\operatorname{Sub}_{1}(\mathfrak{B}, u, v)$ is the set of all coatoms of the lattice $\operatorname{Sub}(\mathfrak{B}, u, v)$ that belong to neither $\operatorname{Sub}(\mathfrak{B}, u)$ nor $\operatorname{Sub}(\mathfrak{B}, v)$.

If $x$ and $y$ are two distinct variables, then $T_{x=y}$ denotes the elementary substitution that swaps $x$ and $y$ and is identical on all other variables.

Lemma 10.11 Let $\mathfrak{B}=\mathfrak{B}_{\ell, r, n}$ for some $\ell, r \geq 0$ and $n>1$ and let $u$ and $v$ be words with $\operatorname{Cont}(u)=\operatorname{Cont}(v)$ and $|\operatorname{Cont}(u)| \geq 3$, of lengths shorter than $|\mathfrak{B}|$, and incomparable in the order $\leq_{\mathfrak{B}}$. If $\ell=r$ [respectively $\ell \neq r$ ], then the variety $\mathcal{V}=\operatorname{var}\{u \approx v, \mathfrak{B}\}$ is definable up to $\operatorname{var}\left\{x^{2} y \approx 0, \mathfrak{B}\right\}$ [respectively definable] in $L(\operatorname{var} \mathfrak{B})$ by the formula: $\mathcal{V} \in \operatorname{Sub}_{1}(\mathfrak{B}, u, v)$ and for every transposition $q$ of $\operatorname{Cont}(v)$ the variety $\operatorname{var}\{u \approx q(u), \mathfrak{B}\}$ contains the variety $\mathcal{V} \wedge \operatorname{var}\{v \approx q(v), \mathfrak{B}\}$. 
Proof If a variety satisfies both $u \approx v$ and $v \approx q(v)$, then, obviously, it satisfies $u \approx$ $q(u)$. Hence, the variety $\mathcal{V}=\operatorname{var}\{u \approx v, \mathfrak{B}\}$ satisfies the proposed formula.

Assume that $\mathcal{V}=\operatorname{var}\{v \approx p(u), \mathfrak{B}\}$ for some permutation $p$ of $\operatorname{Cont}(u)$ and for every transposition $q$ of $\operatorname{Cont}(v)$ the variety $\operatorname{var}\{u \approx q(u), \mathfrak{B}\}$ contains the variety $\mathcal{V} \wedge \operatorname{var}\{v \approx q(v), \mathfrak{B}\}$. We have to show that $p$ is the identity permutation. Suppose, to the contrary, that $p(x)=y$ for some $x \neq y$. Let $z \in \operatorname{Cont}(v) \backslash\{x, y\}$ and take $q=T_{x, z}$. Denote $\mathcal{V}_{1}=\mathcal{V} \wedge \operatorname{var}\left\{v \approx T_{x, z}(v), \mathfrak{B}\right\}=\operatorname{var}\left\{v \approx p(u), v \approx T_{x, z}(v), \mathfrak{B}\right\}$. Since the words $u$ and $v$ are shorter than $|\mathfrak{B}|$ and incomparable in the order $\leq_{\mathfrak{B}}$, we have that $[v] \mathcal{V}_{1}=\left\{v, p(u), T_{x, z}(v), T_{x, z} p(u)\right\}$. By the assumption, $\mathcal{V}_{1} \subseteq\left[u \approx T_{x, z}(u), \mathfrak{B}\right.$. Therefore, $\mathcal{V}_{1}$ satisfies the identity $u \approx T_{x, z}(u)$ and also the identity $p(u) \approx p T_{x, z}(u)$.

Since the words $u$ and $v$ are incomparable in the order $\leq_{\mathfrak{B}}$, the word $p T_{x, z}(u)$ must coincide with either $p(u)$ or $T_{x, z} p(u)$. If $p T_{x, z}(u)=p(u)$ then $p(x)=p(z)$, which contradicts the fact that $p$ is a permutation. If $p T_{x, z}(u)=T_{x, z} p(u)$, then $p(u)=T_{x, z} p T_{x, z}(u)$. But then $T_{x, z} p T_{x, z}(z)=p(x)=y \neq p(z)$, a contradiction. Therefore, $p$ is the identity permutation.

Lemma 10.12 Let $\mathfrak{B}=\mathfrak{B}_{\ell, r, n}$ for some $\ell, r \geq 0$ and $n>1$ and let $u$ and $v$ be words with $\operatorname{Cont}(u)=\operatorname{Cont}(v)=\{x, y\}$, of lengths shorter than $|\mathfrak{B}|$, and incomparable in the order $\leq_{\mathfrak{B}}$. If $\ell=r$ [respectively $\left.\ell \neq r\right]$, then the variety $\mathcal{V}=\operatorname{var}\{u \approx v, \mathfrak{B}\}$ is definable up to $\operatorname{var}\left\{x^{2} y \approx 0, \mathfrak{B}\right\}$ [respectively definable] in $L(\operatorname{var} \mathfrak{B})$ by the formula: $\mathcal{V} \in \operatorname{Sub}_{1}(\mathfrak{B}, u, v)$ and $\mathcal{V} \subset \operatorname{var}\{u z \approx v z, \mathfrak{B}\}$, where $z \notin\{x, y\}$.

Proof Since $|\operatorname{Cont}(v)|=2$, there are only two varieties in the $\operatorname{set} \operatorname{Sub}_{1}(\mathfrak{B}, u, v)$ : $\mathcal{V}=\operatorname{var}\{u \approx v, \mathfrak{B}\}$ and $\operatorname{var}\left\{u \approx T_{x, y}(v), \mathfrak{B}\right\}$. We have to distinguish between them. Since $z \notin\{x, y\}$, we have $|\operatorname{Cont}(u z)|=|\operatorname{Cont}(v z)|=3$. Since the words $u$ and $v$ are incomparable in the order $\leq_{\mathfrak{B}}$, the words $u z$ and $v z$ are also incomparable. Hence, by Lemma 10.11 , the variety $\operatorname{var}\{u z \approx v z, \mathfrak{B}\}$ is semi-definable in $L$ (var $\mathfrak{B})$. Moreover, $u z \approx v z$ is, obviously, a consequence of $u \approx v$, and is not a consequence of $u \approx T_{x, y}(v)$. Therefore, $\mathcal{V} \subset \operatorname{var}\{u z \approx v z, \mathfrak{B}\}$ and $\operatorname{var}\left\{u \approx T_{x, y}(v), \mathfrak{B}\right\} \not \subset \operatorname{var}\{u z \approx$ $v z, \mathfrak{B}\}$. Thus, both $\mathcal{V}$ and $\operatorname{var}\left\{u \approx T_{x, y}(v), \mathfrak{B}\right\}$ are definable up to $\operatorname{var}\left\{x^{2} y \approx 0, \mathfrak{B}\right\}$ [respectively definable] in $L(\operatorname{var} \mathfrak{B})$.

Lemmas 10.11 and 10.12 cover all the cases and complete the proof of Theorem 10.2.

\section{Definability of permutative nil-varieties in $L\left(\operatorname{var} \mathfrak{B}_{\ell, r, n}\right)$}

It is easy to see that every identity $u \approx v$ falls into one of the following four categories.

Case 1: $u \approx v$ is irregular.

Case 2: $u \approx v$ is regular and $u<v$.

Case 3: $v=p(u)$ for some permutation $p$ of $\operatorname{Cont}(u)$.

Case 4: $u \approx v$ is regular and $u$ and $v$ are incomparable in the order $\leq$.

An identity as in Case 3 is called substitutive and an identity as in Case 4 is called parallel. 
Lemma 11.1 Let $\mathfrak{B}$ be a self-dual set of regular identities and $\mathcal{V} \in L$ (var $\mathfrak{B})$. If the variety $\mathcal{V}$ is definable in $L(\operatorname{var} \mathfrak{B})$ up to $\operatorname{var}\left\{x^{2} y \approx 0, \mathfrak{B}\right\}$, then $\mathcal{V}$ is semi-definable in $L(\operatorname{var} \mathfrak{B})$.

Proof Let $\Phi_{\mathcal{V}}(\bar{x}, \bar{y})$ be a first-order formula in two free variables that turns into a true statement on $L(\operatorname{var} \mathfrak{B})$ if and only if $\bar{x}=\mathcal{V}$ and $\bar{y}=\operatorname{var}\left\{x^{2} y \approx 0, \mathfrak{B}\right\}$, or else $\bar{x}=\mathcal{V}^{\delta}$ and $\bar{y}=\operatorname{var}\left\{y x^{2} \approx 0, \mathfrak{B}\right\}$. Then formula $\exists \bar{y} \Phi_{\mathcal{V}}(\bar{x}, \bar{y})$ defines the set $\left\{\mathcal{V}, \mathcal{V}^{\delta}\right\}$ in $L(\operatorname{var} \mathfrak{B})$.

\section{Lemma 11.2 [16]}

(i) For every permutation identity $\tau$ with $\ell(\tau)=0$ and $r(\tau)=0$ there is an $n>1$ such that $\tau \vdash \mathfrak{B}_{0,0, n}$.

(ii) For every two permutation identities $\tau_{1}$ and $\tau_{2}$ with $\ell\left(\tau_{1}\right)=\ell$ and $r\left(\tau_{2}\right)=r$ there is an $n>1$ such that $\left\{\tau_{1}, \tau_{2}\right\} \vdash \mathfrak{B}_{\ell, r, n}$.

(iii) If $\mathcal{V}$ is a permutative variety, then $\ell=\ell(\mathcal{V})$ and $r=r(\mathcal{V})$ are the minimal numbers such that $\mathcal{V} \models \mathfrak{B}_{\ell, r, n}$ for some $n>1$.

Proof (ii) Without loss of generality, we may assume that the identities $\tau_{1}$ and $\tau_{2}$ are written in different variables. Since $\ell\left(\tau_{1}\right)=\ell$, we have that $t_{1} t_{2} \cdots t_{\ell} u \approx t_{1} t_{2} \cdots t_{\ell} u^{\prime}$ such that the words $u$ and $u^{\prime}$ begin with different variables. Since $r\left(\tau_{1}\right)=r$, we have that $v z_{1} z_{2} \cdots z_{r} \approx v^{\prime} z_{1} z_{2} \cdots z_{r}$ such that the words $v$ and $v^{\prime}$ end with different variables. These two identities imply the identity

$$
t_{1} t_{2} \cdots t_{\ell} u v z_{1} z_{2} \cdots z_{r} \approx t_{1} t_{2} \cdots t_{\ell} u^{\prime} v^{\prime} z_{1} z_{2} \cdots z_{r}
$$

By the result of Putcha and Yaqub (part (i)) the identity $u v \approx u^{\prime} v^{\prime}$ implies all identities in $\mathfrak{B}_{0,0, n}$ for some $n>1$. Therefore, by using the identity (1) we can derive all identities in $\mathfrak{B}_{\ell, r, n}$.

(iii) Follows immediately from part (ii) and the definition of the functions $\ell=\ell(\mathcal{V})$ and $r=r(\mathcal{V})$.

Theorem 11.1 For each permutative nil-variety $\mathcal{V}$ and each $\ell \geq \ell(\mathcal{V})$ and $r \geq r(\mathcal{V})$ there exists $n>1$ such that $\mathcal{V}$ is definable in $L\left(\operatorname{var} \mathfrak{B}_{\ell, r, n}\right)$ if $\ell \neq r$ or $\mathcal{V}$ is semidefinable in $L\left(\operatorname{var} \mathfrak{B}_{\ell, r, n}\right)$ if $\ell=r$.

Proof If $\mathcal{V}$ is a permutative nil-variety, then by a result of Perkins [15], we have that $\mathcal{V}=\operatorname{var} \Sigma$ for some finite set of identities $\Sigma$. Let $n$ be bigger than the lengths of all identities in $\Sigma$. By Lemma 11.2, for each $\ell \geq \ell(\mathcal{V})$ and $r \geq r(\mathcal{V})$ we have that $\mathcal{V} \models \mathfrak{B}_{\ell, r, n}$. Therefore, we may assume that $\Sigma$ contains the set of identities $\mathfrak{B}_{\ell, r, n}$. Obviously, in a nil-variety, every irregular identity $u \approx v$ is equivalent to the pair of identities $u \approx 0$ and $v \approx 0$. By Lemma 7.8, every identity $u \approx v$ as in Case 2 is also equivalent to $u \approx 0$ and $v \approx 0$. Therefore, we may assume that in addition to $\mathfrak{B}_{\ell, r, n}$ the set $\Sigma$ also contains only 0 -reduced identities, substitutive identities and parallel identities. Theorems 9.1, 10.1 and 10.2 and Lemmas 9.2 and 11.1 imply that $\mathcal{V}$ is definable in $L\left(\operatorname{var} \mathfrak{B}_{\ell, r, n}\right)$ if $\ell \neq r$ and semi-definable in $L\left(\operatorname{var} \mathfrak{B}_{\ell, r, n}\right)$ if $\ell=r$. 
Lemma 11.3 If $u \approx v$ is a regular identity such that $u<v$ then $|u|<|v|$.

Proof Since $u \leq v$, we have that $v=a \Theta(u) b$ for some possibly empty words $a$ and $b$ and some substitution $\Theta$. If one of the words $a$ or $b$ is not empty or the value of $\Theta$ on one of the variables is not a one-letter word, then the word $v$ is longer than $u$. So, we may assume that both words $a$ and $b$ are empty and that substitution $\Theta$ maps the variables to one-letter words. Since the identity $u \approx v$ is regular, the substitution $\Theta$ maps the variables in $u$ to some variables in $\operatorname{Cont}(u)=\operatorname{Cont}(v)$. If any two variables are identified, then the identity $u \approx v$ is irregular. The remaining case is that $u=p(v)$ for some permutation $p$ of $\operatorname{Cont}(u)=\operatorname{Cont}(v)$ which contradicts the fact that $u<v$.

The next lemma follows from Lemma 2 and the proof of Proposition 1 in [28].

Lemma 11.4 [28] Let $\mathcal{V}$ be a periodic 0-permutative variety. Then for $m=m(\mathcal{V})$, $d=d(\mathcal{V})$ and some nil-variety $\mathcal{N}$ we have that $\mathcal{V}=\mathcal{N} \vee \operatorname{var} \mathbf{C}_{m, d}$

Corollary 11.1 ([3], Theorem 10.9) Every 0-permutative variety is semi-definable in the lattice $\mathbf{P}(\mathbf{0})$ of all 0-permutative varieties.

Proof If $\mathcal{V}$ is a 0 -permutative variety, then $\ell(\mathcal{V})=r(\mathcal{V})=0$ and by the result of Putcha and Yaqub [16] (see Lemma 11.2), the variety $\mathcal{V}$ is finitely based.

If $\mathcal{V}$ is periodic, then by Lemma $11.4, \mathcal{V}$ is a join of a nil-variety $\mathcal{N}$ and $\operatorname{var}\left(\mathbf{C}_{m, d}\right)$. Since $\mathcal{N}$ is semi-definable in $L\left(\operatorname{var} \mathfrak{B}_{0,0, n}\right)$ for some $n>1$ by Theorem 11.1 , the variety $\mathcal{V}$ is semi-definable in $L\left(\operatorname{var} \mathfrak{B}_{0,0, n}\right)$ by Lemmas 2.2 and 2.3. Therefore, $\mathcal{V}$ is semi-definable in $\mathbf{P}(\mathbf{0})$ by Lemma 2.7 .

If $\mathcal{V}$ is not periodic, then in view of Lemma 11.3 and Proposition $3.1, \mathcal{V}$ satisfies only substitutive and/or parallel identities. In this case, Theorems 10.1 and 10.2 and Lemmas 9.2 and 11.1 imply that $\mathcal{V}$ is semi-definable in $L\left(\operatorname{var} \mathfrak{B}_{0,0, n}\right)$ for some $n$ big enough. Now Lemma 2.7 implies that $\mathcal{V}$ is semi-definable in $\mathbf{P}(\mathbf{0})$.

For each $k>0$, denote $\mathcal{L Z}(k)=\operatorname{var}\left\{t_{1} t_{2} \cdots t_{k} \approx t_{1} t_{2} \cdots t_{k} x\right\}$ and $\mathcal{R Z}(k)=$ $\operatorname{var}\left\{t_{1} t_{2} \cdots t_{k} \approx x t_{1} t_{2} \cdots t_{k}\right\}$. Note that $\mathcal{L} \mathcal{Z}=\mathcal{L} \mathcal{Z}(1)$ and $\mathcal{R} \mathcal{Z}=\mathcal{R} \mathcal{Z}(1)$ are the only non-commutative atoms of SEM.

Conjecture 11.1 For each $k>0$ the variety $\mathcal{L} \mathcal{Z}(k)$ is semi-definable in every selfdual ideal I of SEM that contains $\mathcal{L} \mathcal{Z}(k)$ and $\operatorname{var}\{x y \approx y x\}$.

Since the variety $\mathcal{L} \mathcal{Z}(k)$ is given by an irregular identity, Conjecture 11.1 is true for $\mathbf{I}=\mathbf{S E M}$ by [11, Proposition 8.2].

Lemma 11.5 Conjecture 11.1 is true for $k<3$.

Proof If $k=1$, then it is proved in [11, Lemma 4.3] and reproved in [25, Proposition 1.4] that the set $\{\mathcal{L Z}, \mathcal{R Z}\}$ is definable in SEM. The arguments in [25, Proposition 1.4] (see the explanations after Lemma 2.1) can be used to show that the set $\{\mathcal{L}, \mathcal{R Z}\}$ is definable in $\mathbf{I}$. 
Here are the arguments of Vernikov that show that the set $\{\mathcal{L Z}(2), \mathcal{R} \mathcal{Z}(2)\}$ is definable in $\mathbf{I}$.

For each $k>0$ we have

$$
\begin{aligned}
& \operatorname{var}\left\{t_{1} t_{2} \cdots t_{k} y \approx t_{1} t_{2} \cdots t_{k} x\right\} \\
& \quad=\operatorname{var}\left\{t_{1} t_{2} \cdots t_{k} \approx t_{1} t_{2} \cdots t_{k} x\right\} \vee \operatorname{var}\left\{x_{1} x_{2} \cdots x_{k} x_{k+1} \approx 0\right\}
\end{aligned}
$$

So, the variety $\operatorname{var}\{t x \approx t y\}=\mathcal{L} \mathcal{Z} \vee \mathcal{Z} \mathcal{M}$ is semi-definable in $\mathbf{I}$.

Now the variety $\mathcal{L} \mathcal{Z}(2)=\operatorname{var}\left\{t_{1} t_{2} \approx t_{1} t_{2} x\right\}$ is the only cover of the variety $\operatorname{var}\{t x \approx t y\}$ with the following properties.

1. $\mathcal{L} \mathcal{Z}(2)$ does not contain any atoms of $\mathbf{I}$ but $\mathcal{L} \mathcal{Z}$ and $\mathcal{Z} \mathcal{M}$.

2. $\mathcal{L} \mathcal{Z}(2)$ does not contain $\mathcal{N}_{3}$.

Lemma 11.6 For each $k \geq 0, n>1$ and each $\ell, r$ with $0 \leq \ell, r \leq \min (k, 2)$ the set $\left\{\operatorname{var} \mathfrak{B}_{\ell, r, n}, \operatorname{var} \mathfrak{B}_{r, \ell, n}\right\}$ is definable in the lattice $\mathbf{P}(\mathbf{k})$ of all $k$-permutative varieties.

Proof In view of Lemma 2.7 we may assume that $k>0$. It is easy to check that $\operatorname{var} \mathfrak{B}_{\ell, r, n}=\operatorname{var} \mathfrak{B}_{0,0, n+l+r} \vee \mathcal{L} \mathcal{Z}(\ell) \vee \mathcal{R} \mathcal{Z}(r)$. In view of Lemmas 2.7 and 11.5, the set $\left\{\operatorname{var} \mathfrak{B}_{\ell, r, n}\right.$, var $\left.\mathfrak{B}_{r, \ell, n}\right\}$ is definable in $\mathbf{P}(\mathbf{k})$.

The difficulty of extending Lemma 11.6 to arbitrary $\ell, r$ with $0 \leq \ell, r \leq k$ lies in extending Lemma 11.5 to $k>2$.

Theorem 11.1 and Lemma 11.6 imply the following.

Corollary 11.2 For each $k=0,1,2$, every $k$-permutative nil-variety is semidefinable in the lattice $\mathbf{P}(\mathbf{k})$ of all $k$-permutative varieties.

Acknowledgements O. Sapir thanks Dr. Ralph McKenzie for directing her attention to the articles [2, 3] and Dr. Boris Vernikov for teaching her this subject and for many illuminating discussions. The authors are also grateful to Vyacheslav Shaprynskii for finding a hole in the previous version.

Open Access This article is distributed under the terms of the Creative Commons Attribution License which permits any use, distribution, and reproduction in any medium, provided the original author(s) and the source are credited.

\section{References}

1. Evans, T.: The lattice of semigroup varieties. Semigroup Forum 2, 1-43 (1971)

2. Grech, M.: Automorphisms of the lattice of equational theories of commutative semigroups. Trans. Am. Math. Soc. 361, 3435-3462 (2009)

3. Grech, M.: The structure and definability in the lattice of equational theories of strongly permutative semigroups. Trans. Am. Math. Soc. 364, 2959-2985 (2012)

4. Ježek, J.: Intervals in lattices of varieties. Algebra Univers. 6, 147-158 (1976)

5. Ježek, J.: The lattice of equational theories I: modular elements. Czechoslov. Math. J. 31, 127-152 (1981)

6. Ježek, J.: The lattice of equational theories II: the lattice of full sets of terms. Czechoslov. Math. J. 31, 573-603 (1981)

7. Ježek, J.: The lattice of equational theories III: definability and authomorphisms. Czechoslov. Math. J. 32, 129-164 (1982) 
8. Ježek, J.: The lattice of equational theories IV: equational theories of finite algebras. Czechoslov. Math. J. 36, 331-341 (1986)

9. Ježek, J.: The ordering of commutative terms. Czechoslov. Math. J. 56, 133-154 (2006)

10. Ježek, J.: Definability for equational theories of commutative groupoids. Czechoslov. Math. J. 62, 305-333 (2012)

11. Jezek, J., McKenzie, R.: Definability in the lattice of equational theories of semigroups. Semigroup Forum 46, 199-245 (1993)

12. Kisielewicz, A.: Definability in the lattice of equational theories of commutative semigroups. Trans. Am. Math. Soc. 356, 3483-3504 (2004)

13. McKenzie, R.: Definability in the lattice of equational theories. Ann. Math. Log. 3, 197-237 (1971)

14. Martynova, T.: The groupoid of 0-reduced semigroup varieties. Ural. Gos. Univ. Mat. Zap. 11(3), 96-115 (1979) (Russian)

15. Perkins, P.: Bases for equational theories of semigroups. J. Algebra 11, 298-314 (1969)

16. Putcha, M., Yaqub, A.: Semigroups satisfying permutation identities. Semigroup Forum 3, 68-73 (1971)

17. Sapir, O.: Finitely generated permutative varieties. Semigroup Forum 78, 427-449 (2009)

18. Shaprynskii, V.: Distributive and neutral elements of the lattice of commutative semigroup varieties. Izv. Vysš. Učebn. Zaved., Mat. 7(7), 67-79 (2011) (Russian; Engl. translation in Russ. Math. (Iz. VUZ), 55, 56-5647 (2011))

19. Shaprynskii, V.: Modular and lower-modular elements of lattices of semigroup varieties. Semigroup Forum 85, 97-110 (2012)

20. Shevrin, L., Volkov, M.: Identities of semigroups. Izv. Vysš. Učebn. Zaved., Mat. 11(11), 3-47 (1985) (Russian; Engl. translation in J. Sov. Math. (Iz. VUZ) 29, 1-64 (1985))

21. Subramanian, H., Sundararaman, T.: Precomplete varieties of semigroups. Semigroup Forum 8, 278281 (1974)

22. Tarski, A.: Equational logic and equational theories of algebras. In: Contributions to Math. Logic, Colloquium, Hannover, 1966, pp. 275-288. North-Holland, Amsterdam (1968)

23. Vernikov, B.: Semimodular and Arguesian varieties of semigroups: forbidden subvarieties. Proc. Ural State Univers. 22(4), 16-42 (2002) (Russian)

24. Vernikov, B.: Lower-modular elements of the lattice of semigroup varieties. Semigroup Forum 75, 554-566 (2007)

25. Vernikov, B.: Proofs of definability of some varieties and sets of varieties of semigroups. Semigroup Forum 84, 374-392 (2012)

26. Vernikov, B.: Definability of the Variety Generated by a Commutative Monoid in the Lattice of Commutative Semigroups Varieties. Preprint, available under arXiv:1011.1557v3 [math.GR]

27. Vernikov, B., Volkov, M.: Commuting fully invariant congruences on free semigroups. Contrib. Gen. Algebra 12, 391-417 (2000)

28. Volkov, M.: Semigroup varieties with modular subvariety lattices. Izv. Vysš. Učebn. Zaved., Mat. 6(6), 51-60 (1989) (Russian; Engl. translation in J. Sov. Math. (Iz. VUZ) 33, 48-58 (1989))

29. Volkov, M.: Modular elements in the lattice of semigroup varieties. Contrib. Gen. Algebra 16, 275$288(2005)$ 